\title{
A Rhodium(I)-Catalyzed Formal Allenic Alder-ene Reaction for the Rapid and Stereoselective Assembly of Cross-Conjugated Trienes.
}

Kay M. Brummond*, Hongfeng Chen, Peter Sill and Lingfeng You.

Department of Chemistry, University of Pittsburgh, Pittsburgh, Pennsylvania

\section{Experimental Section}
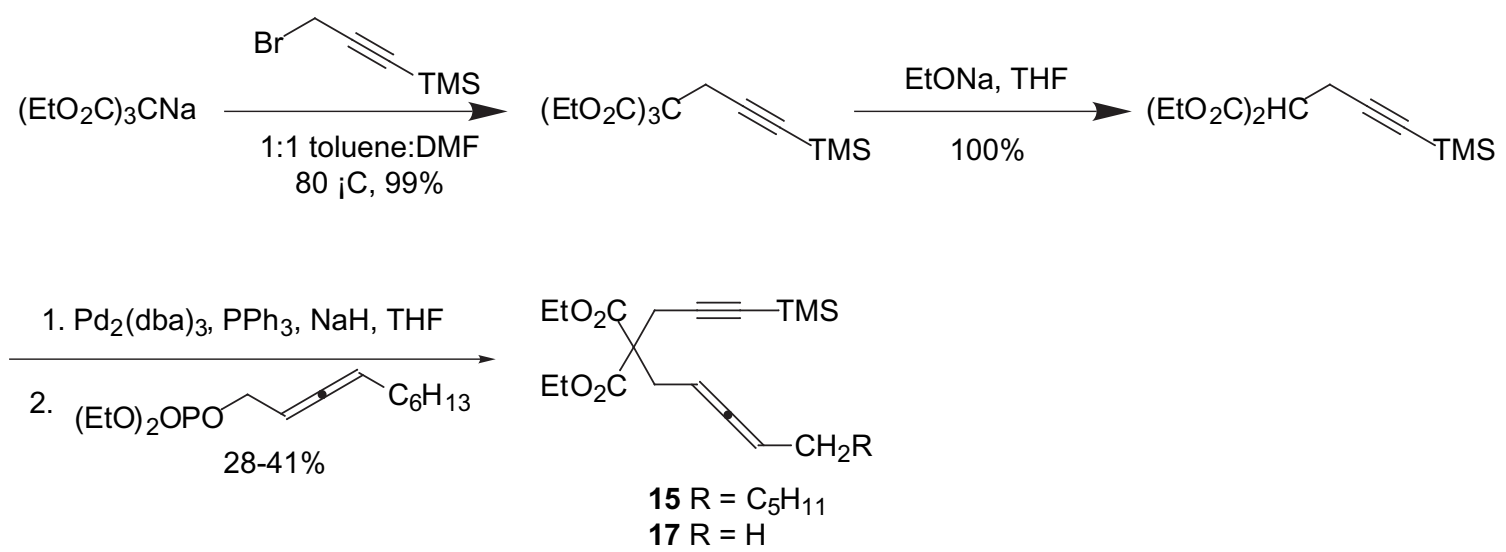

2-(2,3-Decadienyl)-2-(3-trimethylsilanyl-2-propynyl)malonic acid diethyl ester (15). A solution of 2-(3-trimethylsilyl-2-propynyl )malonic acid diethyl ester (0.29 $\mathrm{g}, 1.1 \mathrm{mmol})$ in THF $(2 \mathrm{~mL})$ was added dropwise to a suspension of $\mathrm{NaH}(44 \mathrm{mg}, 60 \%$ dispersion in mineral oil, $1.1 \mathrm{mmol})$ in THF $(10 \mathrm{~mL})$. The resulting clear solution was stirred at rt for $20 \mathrm{~min}$ and $\mathrm{PPh}_{3}(0.14 \mathrm{~g}, 0.26 \mathrm{mmol})$ was added. The flask was flushed with a stream of $\mathrm{N}_{2}$ gas for $5 \mathrm{~min} \mathrm{Pd}_{2}(\mathrm{dba})_{3}(40 \mathrm{mg}, 0.044 \mathrm{mmol})$ and phosphoric acid 2,3-decadienyl ester diethyl ester $(0.30 \mathrm{~g}, 1.1 \mathrm{mmol})$ were added at $0 \nmid \mathrm{C}$. The mixture was stirred at $0 ; \mathrm{C}$ for $2 \mathrm{~h}$ and quenched with sat. $\mathrm{NH}_{4} \mathrm{Cl}$ solution $(5 \mathrm{~mL})$. The resulting mixture was extracted with ether, and the combined ether layers were washed with brine, dried $\left(\mathrm{MgSO}_{4}\right)$, and concentrated in vacuo to afford a yellow oil that was purified by flash chromatography $\left(\mathrm{SiO}_{2}\right.$, pentane : diethyl ether $\left.=30: 1\right)$ to afford title compound 15 as a colorless oil $(0.187 \mathrm{~g}, 42 \%):{ }^{1} \mathrm{H}$ NMR $\left(300 \mathrm{MHz}, \mathrm{CDCl}_{3}\right) \delta$ 5.08-5.02 (m, $\left.1 \mathrm{H}\right)$, 4.91-4.82 (m, $1 \mathrm{H}), 4.25-4.11(\mathrm{~m}, 4 \mathrm{H}), 2.85$ (s, $2 \mathrm{H}), 2.71(\mathrm{dd}, J=2.3,7.8 \mathrm{~Hz}, 2 \mathrm{H})$, 1.98-1.90 (m, $2 \mathrm{H}), 1.39-1.19(\mathrm{~m}, 14 \mathrm{H}), 0.86(\mathrm{t}, J=7.0 \mathrm{~Hz}), 0.10(\mathrm{~s}, 9 \mathrm{H}) ;{ }^{13} \mathrm{C} \mathrm{NMR}(75$ $\mathrm{MHz}, \mathrm{CDCl} 3) \delta 205.9,169.7,101.5,91.0,88.0,84.5,61.5,57.3,32.4,31.7,29.2,28.8$, 
23.9, 22.6, 14.1, -0.1; IR (neat) $v$ 1738, 1963, 2180, 2959, $2929 \mathrm{~cm}^{-1}$; EI-HRMS calcd for $\mathrm{C}_{23} \mathrm{H}_{38} \mathrm{O}_{4} \mathrm{Si}\left[\mathrm{M}^{+}\right] \mathrm{m} / \mathrm{z} 406.2539$, found 406.2537.

\section{2-(2,3-Pentadienyl)-2-(3-trimethylsilanyl-2-propynyl)malonic acid diethyl} ester (17). 3-trimethylsilylpropargyl bromide (3.0 g, $15.7 \mathrm{mmol})$ was added to a solution of triethyl sodiomethanetricarboxylate $(4.0 \mathrm{~g}, 15.7 \mathrm{mmol})$ in toluene : DMF $(1: 1,50$ $\mathrm{mL}$ ) and the mixture was stirred and heated at $80 ¡ \mathrm{C}$ overnight. The resulting yellow suspension was cooled to $\mathrm{rt}$, diluted with toluene $(100 \mathrm{~mL})$, washed with water, sat. $\mathrm{Na}_{2} \mathrm{CO}_{3}$ solution, and brine. The combined aqueous layers were back-extracted with toluene and the combined organic layers were dried $\left(\mathrm{MgSO}_{4}\right)$, filtered and concentrated to afford 2-ethoxycarbonyl-2-(3-trimethylsilanyl-2-propynyl)malonic acid diethyl ester as a yellow oil (5.35 g, 99\%).

This oil was dissolved in THF $(20 \mathrm{~mL})$ and added dropwise to a suspension of sodium ethoxide $(1.30 \mathrm{~g}, 19.1 \mathrm{mmol})$ in THF $(30 \mathrm{~mL})$. After stirring at $\mathrm{rt}$ for $2 \mathrm{~h}$ the solution was acidified with $1 \mathrm{~N} \mathrm{HCl}$ and diluted with ether $(150 \mathrm{~mL})$, washed with water $(30 \mathrm{~mL})$, sat. $\mathrm{NaHCO}_{3}$ solution, brine, dried $\left(\mathrm{MgSO}_{4}\right)$, concentrated and purified by flash chromatography $\left(\mathrm{SiO}_{2}\right.$, hexanes : diethyl ether $\left.=3: 1\right)$ to afford 2-(3-trimethylsilanyl-2propynyl)malonic acid diethyl ester as a pale yellow oil (4.15 g, 100\%).

A solution of 2-(3-trimethylsilyl-2-propynyl)malonic acid diethyl ester $(0.14 \mathrm{~g}$, $0.50 \mathrm{mmol})$ in THF $(2 \mathrm{~mL})$ was added dropwise to a suspension of $\mathrm{NaH}(20 \mathrm{mg}, 60 \%$ dispersion in mineral oil, $0.50 \mathrm{mmol})$ in THF $(5 \mathrm{~mL})$. The resulting clear solution was stirred at $\mathrm{rt}$ for $20 \mathrm{~min}$ and $\mathrm{PPh}_{3}(0.032 \mathrm{~g}, 0.12 \mathrm{mmol})$ was added. The flask was flushed with a stream of $\mathrm{N}_{2}$ gas for $5 \mathrm{~min} \mathrm{Pd}_{2}(\mathrm{dba})_{3}(9.2 \mathrm{mg}, 0.01 \mathrm{mmol})$ and phosphoric acid diethyl ester 2,3-pentadienyl ester $(0.11 \mathrm{~g}, 0.51 \mathrm{mmol})$ was added at 0 ¡C . The mixture was stirred at $0 \nmid \mathrm{C}$ for $2 \mathrm{~h}$ and quenched with sat. $\mathrm{NH}_{4} \mathrm{Cl}$ solution. The resulting mixture was extracted with ether, and the combined ether layers were washed with brine, dried $\left(\mathrm{MgSO}_{4}\right)$, and concentrated in vacuo to afford a yellow oil that was purified by flash chromatography $\left(\mathrm{SiO}_{2}\right.$, pentane : diethyl ether $\left.=10: 1\right)$ to afford title compound 17 as a colorless oil (83 mg, 49\%): ${ }^{1} \mathrm{H}$ NMR $\left(300 \mathrm{MHz}, \mathrm{CDCl}_{3}\right) \delta$ 5.05-5.01 (m, $\left.1 \mathrm{H}\right), 4.89-4.82$ (m, $1 \mathrm{H}), 4.23-4.12(\mathrm{~m}, 4 \mathrm{H}), 2.85(\mathrm{~s}, 2 \mathrm{H}), 2.71(\mathrm{dd}, J=2.2,7.8 \mathrm{~Hz}, 2 \mathrm{H}), 1.60$ (dd, $J=$ 3.2, 7.0 Hz, $3 \mathrm{H}), 1.25$ (t, $J=7.1 \mathrm{~Hz}, 6 \mathrm{H}), 0.10(\mathrm{~s}, 9 \mathrm{H}) ;{ }^{13} \mathrm{C}$ NMR $(75 \mathrm{MHz}, \mathrm{CDCl} 3) \delta$ $206.8,169.7,101.4,87.9,85.5,84.0,61.5,57.3,32.2,23.9,14.2,14.0,-0.1$; IR (neat) $v$ 
1738, 1963, 2180, 2960, $2928 \mathrm{~cm}^{-1}$; EI-HRMS calcd for $\mathrm{C}_{18} \mathrm{H}_{28} \mathrm{O}_{4} \mathrm{Si}\left[\mathrm{M}^{+}\right] \mathrm{m} / z$ 336.1757, found 336.1770 .
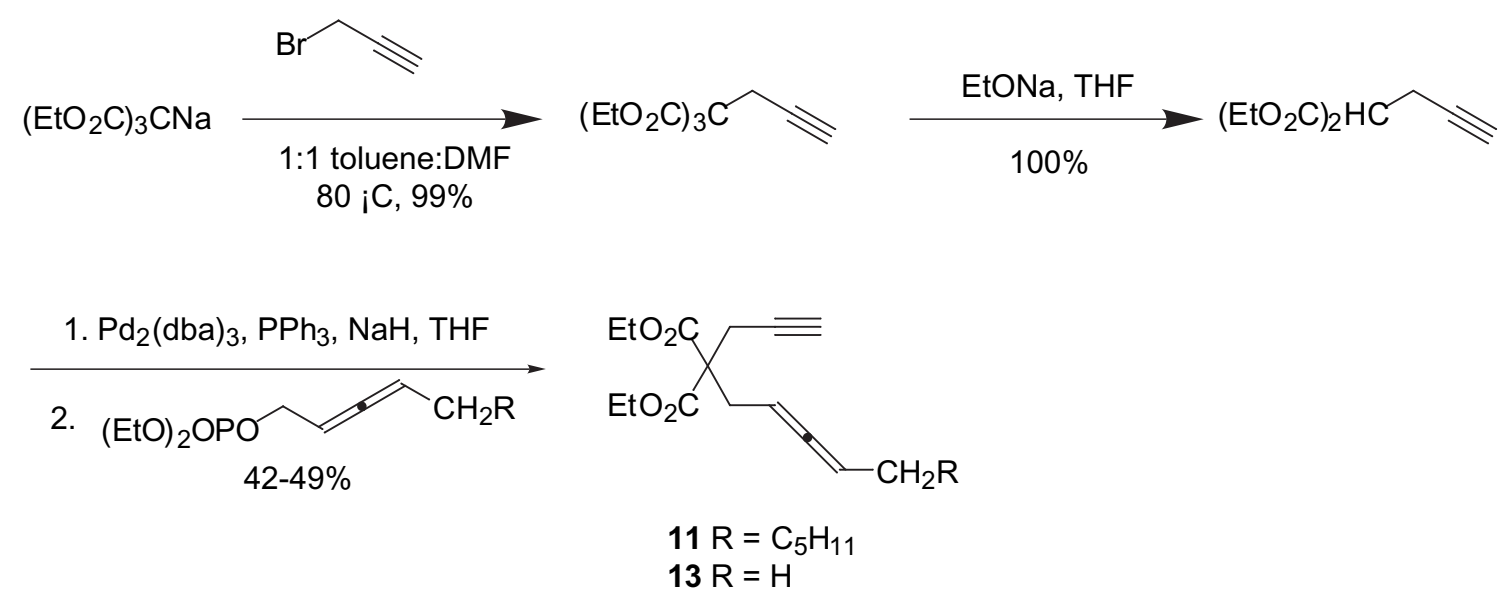

2-(2,3-Decadienyl)-2-(2-propynyl)malonic acid diethyl ester (11). A solution of 2-(2-propynyl)malonic acid diethyl ester $(0.22 \mathrm{~g}, 1.1 \mathrm{mmol})$ in THF $(2 \mathrm{~mL})$ was added dropwise to a suspension of $\mathrm{NaH}(44.0 \mathrm{mg}, 60 \%$ dispersion in mineral oil, $1.1 \mathrm{mmol})$ in THF $(5 \mathrm{~mL})$. The resulting clear solution was stirred at $\mathrm{rt}$ for $20 \mathrm{~min}$ and $\mathrm{PPh}_{3}(0.14 \mathrm{~g}$, $0.260 \mathrm{mmol}$ ) was added. The flask was flushed with a stream of $\mathrm{N}_{2}$ gas for $5 \mathrm{~min}$ $\mathrm{Pd}_{2}(\mathrm{dba})_{3}(40 \mathrm{mg}, 0.04 \mathrm{mmol})$ and phosphoric acid 2,3-decadienyl ester diethyl ester $(0.300 \mathrm{~g}, 1.10 \mathrm{mmol})$ was added at $0 \nmid \mathrm{C}$. The mixture was stirred at $0{ }_{i} \mathrm{C}$ for $2 \mathrm{~h}$ and quenched with sat. $\mathrm{NH}_{4} \mathrm{Cl}$ solution. The resulting mixture was extracted with ether, and the combined ether layers were washed with brine, dried $\left(\mathrm{MgSO}_{4}\right)$, and concentrated in vacuo to afford a yellow oil that was purified by flash chromatography $\left(\mathrm{SiO}_{2}\right.$, pentane : diethyl ether $=10: 1)$ to afford title compound $\mathbf{1 1}$ as a colorless oil $(0.148 \mathrm{~g}, 41 \%):{ }^{1} \mathrm{H}$ NMR (300 MHz, $\left.\mathrm{CDCl}_{3}\right) \delta$ 5.09-5.02 (m, $\left.1 \mathrm{H}\right), 4.90-4.82(\mathrm{~m}, 1 \mathrm{H}), 4.26-4.11(\mathrm{~m}, 4 \mathrm{H})$, $2.83(\mathrm{~d}, J=2.7 \mathrm{~Hz}, 2 \mathrm{H}), 2.72(\mathrm{dd}, J=2.3,7.8 \mathrm{~Hz}, 2 \mathrm{H}), 1.98-1.90$ (m, $2 \mathrm{H}), 1.38-1.18$ $(\mathrm{m}, 8 \mathrm{H}), 1.23(\mathrm{t}, J=7.1 \mathrm{~Hz}, 6 \mathrm{H}), 0.86(\mathrm{t}, J=7.0 \mathrm{~Hz}, 3 \mathrm{H}) ;{ }^{13} \mathrm{C} \mathrm{NMR}(75 \mathrm{MHz}, \mathrm{CDCl})$ $\delta$ 206.0, 169.7, 91.2, 84.3, 79.0, 71.2, 61.6, 57.0, 32.3, 31.7, 29.1, 28.8, 22.6, 22.5, 14.0; IR (neat) $v 1736,1963,3293 \mathrm{~cm}^{-1}$; EI-HRMS calcd for $\mathrm{C}_{20} \mathrm{H}_{30} \mathrm{O}_{4}\left[\mathrm{M}^{+}\right] \mathrm{m} / z$ 334.2144, found 334.2146 .

2-(2,3-Pentadienyl)-2-(2-propynyl)malonic acid diethyl ester (13). Propargyl bromide $(2.85 \mathrm{~mL}, 80 \%$ solution in toluene, $9.50 \mathrm{mmol})$ was added to a solution of 
triethyl sodiomethanetricarboxylate $(2.42 \mathrm{~g}, 9.50 \mathrm{mmol})$ in toluene : DMF $(1: 1,24 \mathrm{~mL})$ and the mixture was stirred and heated at $80 ; \mathrm{C}$ overnight. The resulting yellow suspension was cooled to rt, diluted with toluene $(100 \mathrm{~mL})$, washed with water, sat. $\mathrm{Na}_{2} \mathrm{CO}_{3}$ solution, and brine $(2 \mathrm{X})$. The combined aqueous layers were back-extracted with toluene and the combined organic layers were dried $\left(\mathrm{MgSO}_{4}\right)$, filtered and concentrated to afford 2-ethoxycarbonyl-2-(2-propynyl)malonic acid diethyl ester as a yellow oil (2.53 g, 100\%).

This oil was dissolved in THF (6 mL) and added dropwise to a suspension of sodium ethoxide $(0.800 \mathrm{~g}, 11.8 \mathrm{mmol})$ in THF $(20 \mathrm{~mL})$. After stirring at $\mathrm{rt}$ for $2 \mathrm{~h}$ the solution was acidified with $1 \mathrm{~N} \mathrm{HCl}$ and diluted with ether $(150 \mathrm{~mL})$, washed with water $(30 \mathrm{~mL})$, sat. $\mathrm{NaHCO}_{3}$ solution, brine, dried $\left(\mathrm{MgSO}_{4}\right)$, concentrated and purified by flash chromatography $\left(\mathrm{SiO}_{2}\right.$, hexanes : diethyl ether $\left.=3: 1\right)$ to afford 2-(2-propynyl)malonic acid diethyl ester as a pale yellow oil (1.76 g, 95\%).

A solution of 2-(2-propynyl)malonic acid diethyl ester $(0.136 \mathrm{~g}, 0.503 \mathrm{mmol})$ in THF (2 mL) was added dropwise to a suspension of $\mathrm{NaH}(20 \mathrm{mg}, 60 \%$ dispersion in mineral oil, $0.503 \mathrm{mmol})$ in THF $(5 \mathrm{~mL})$. The resulting clear solution was stirred at $\mathrm{rt}$ for $20 \mathrm{~min}$ and $\mathrm{PPh}_{3}(16 \mathrm{mg}, 0.06 \mathrm{mmol})$ was added. The flask was flushed with a stream of $\mathrm{N}_{2}$ gas for $5 \mathrm{~min} \mathrm{Pd}_{2}(\mathrm{dba})_{3}(9 \mathrm{mg}, 0.01 \mathrm{mmol})$ and phosphoric acid diethyl ester 2,3pentadienyl ester $(0.11 \mathrm{~g}, 0.51 \mathrm{mmol})$ was added at $0 \nmid \mathrm{C}$. The mixture was stirred at $0{ }_{i} \mathrm{C}$ for $2 \mathrm{~h}$ and quenched with sat. $\mathrm{NH}_{4} \mathrm{Cl}$ solution. The resulting mixture was extracted with ether, and the combined ether layers were washed with brine, dried $\left(\mathrm{MgSO}_{4}\right)$, and concentrated in vacuo to afford a yellow oil that was purified by flash chromatography $\left(\mathrm{SiO}_{2}\right.$, pentane $:$ diethyl ether $\left.=10: 1\right)$ to afford title compound $\mathbf{1 3}$ as a colorless oil $(37.7$ mg, 28\%) : ${ }^{1} \mathrm{H}$ NMR (300 MHz, $\left.\mathrm{CDCl}_{3}\right) \delta$ 5.05-5.01 (m, $\left.1 \mathrm{H}\right), 4.88-4.82(\mathrm{~m}, 1 \mathrm{H}), 4.25-$ $4.10(\mathrm{~m}, 4 \mathrm{H}), 2.82(\mathrm{~d}, J=2.7 \mathrm{~Hz}, 2 \mathrm{H}), 2.71(\mathrm{dd}, J=2.2,7.8 \mathrm{~Hz}, 2 \mathrm{H}), 1.96(\mathrm{t}, J=2.7$ $\mathrm{Hz}, 1 \mathrm{H}), 1.63(\mathrm{dd}, J=3.2,7.0 \mathrm{~Hz}, 3 \mathrm{H}), 1.22(\mathrm{t}, J=7.1 \mathrm{~Hz}, 6 \mathrm{H}) ;{ }^{13} \mathrm{C}$ NMR $(75 \mathrm{MHz}$, CDC13) $\delta 206.8,169.6,85.7,83.9,78.9,71.2,61.6,57.0,32.1,22.5,14.2,14.0$; IR (neat) $v$ 1736, 1967, $3287 \mathrm{~cm}^{-1}$; EI-HRMS calcd for $\mathrm{C}_{15} \mathrm{H}_{20} \mathrm{O}_{4}\left[\mathrm{M}^{+}\right] \mathrm{m} / z$ 264.1362, found 264.1364 . 

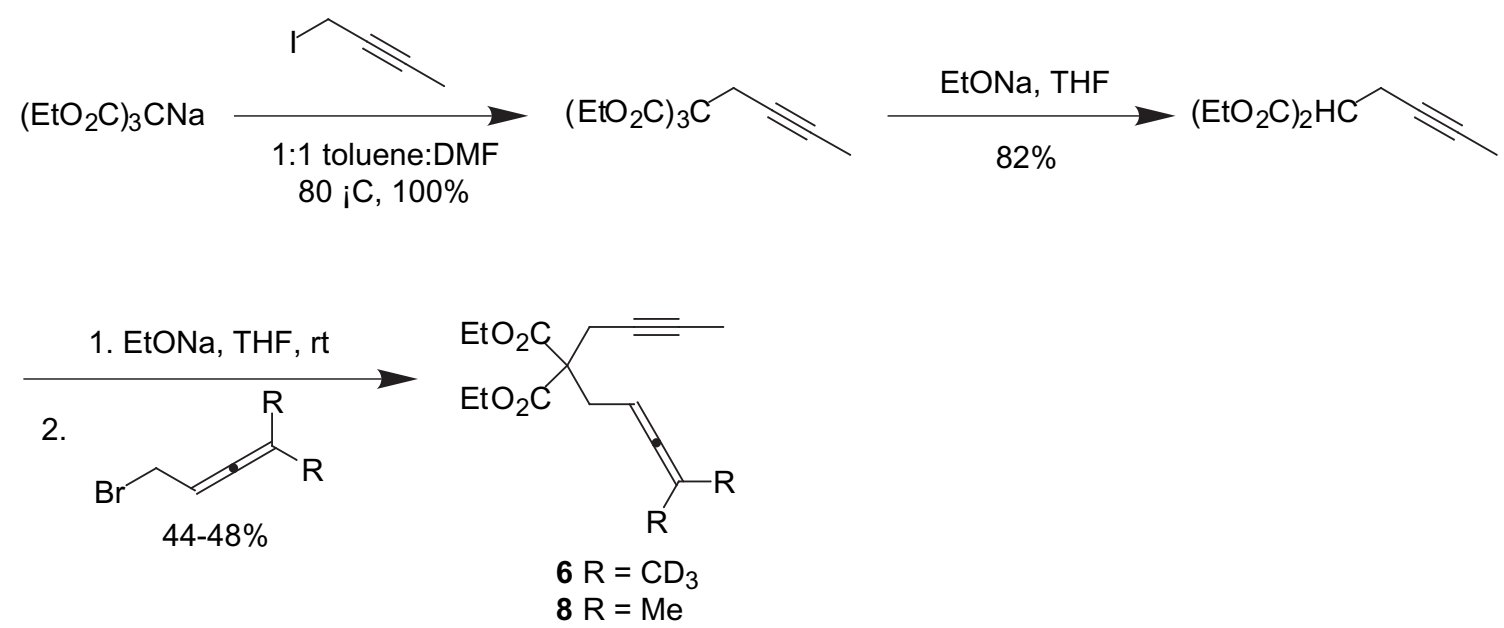

\section{2-(2-Butynyl)-2-(5,5,5-trideuterio-4-trideuteriomethyl-2,3-}

pentadienyl)malonic acid diethyl ester (6). A solution of 2-(2-butynyl)malonic acid diethyl ester $(0.390 \mathrm{~g}, 2.00 \mathrm{mmol})$ in THF $(2 \mathrm{~mL})$ was added dropwise to a suspension of $\mathrm{NaH}(89.0 \mathrm{mg}, 60 \%$ dispersion in mineral oil, $2.02 \mathrm{mmol})$ in THF $(6 \mathrm{~mL})$. The resulting clear solution was stirred at $\mathrm{rt}$ for $20 \mathrm{~min}$ and 5-bromo-1,1,1-trideuterio-2trideuteriomethyl-2,3-pentadiene [prepared by bromination of 5,5,5-trideuterio-4trideuteriomethyl-2,3-pentadien-1-ol (0.4 g, $3.8 \mathrm{mmol})$ with $\mathrm{CBr}_{4}(1.4 \mathrm{~g}, 4.2 \mathrm{mmol})$ and $\mathrm{PPh}_{3}(1.11 \mathrm{~g}, 4.2 \mathrm{mmol})$ in $\mathrm{CH}_{2} \mathrm{Cl}_{2}$ at $\left.0{ }_{i} \mathrm{C}\right]$ was added. The mixture was stirred at $\mathrm{rt}$ overnight and was quenched with sat. $\mathrm{NH}_{4} \mathrm{Cl}$ solution, diluted with ether, washed with brine, sat. $\mathrm{NaHCO}_{3}$ solution, and brine successively, dried $\left(\mathrm{MgSO}_{4}\right)$, concentrated and purified by flash chromatography $\left(\mathrm{SiO}_{2}\right.$, hexanes : diethyl ether $\left.=95: 5\right)$ to afford title compound 6 as a colorless oil $(0.260 \mathrm{~g}, 44 \%):{ }^{1} \mathrm{H} \mathrm{NMR}\left(300 \mathrm{MHz}, \mathrm{CDCl}_{3}\right) \delta 4.78(\mathrm{t}, J=$ $7.6 \mathrm{~Hz}, 1 \mathrm{H}), 4.24-4.13$ (m, $4 \mathrm{H}), 2.78$ (q, $J=2.4 \mathrm{~Hz}, 2 \mathrm{H}), 2.69$ (d, $J=7.6 \mathrm{~Hz}, 2 \mathrm{H}), 1.74$ $(\mathrm{t}, J=2.4 \mathrm{~Hz}, 3 \mathrm{H}), 1.24(\mathrm{t}, J=7.1 \mathrm{~Hz}, 6 \mathrm{H}) ;{ }^{13} \mathrm{C} \mathrm{NMR}(75 \mathrm{MHz}, \mathrm{CDCl} 3) \delta 203.9,170.1$, 94.7, 82.5, 78.4, 73.5, 61.3, 57.4, 32.4, 22.7, 19.5 (multiplet), 14.0, 3.4; IR (neat) v 1736, 1968, $2982 \mathrm{~cm}^{-1}$; EI-HRMS calcd for $\mathrm{C}_{17} \mathrm{H}_{18} \mathrm{D}_{6} \mathrm{O}_{4}\left[\mathrm{M}^{+}\right] \mathrm{m} / z$. 298.2051, found 298.2057.

2-(2-Butynyl)-2-(4-methyl-2,3-pentadienyl)malonic acid diethyl ester (8). 1Iodo-2-butyne $(3.0 \mathrm{~g}, 16.7 \mathrm{mmol})$ was added to a solution of triethyl sodiomethanetricarboxylate $(4.0 \mathrm{~g}, 15.7 \mathrm{mmol})$ in toluene : DMF $(1: 1,40 \mathrm{~mL})$ and the mixture was stirred and heated at $80 ¡ \mathrm{C}$ overnight. The resulting yellow suspension was 
cooled to rt, diluted with ether, washed with water, sat. $\mathrm{Na}_{2} \mathrm{CO}_{3}$ solution, and brine. The combined aqueous layers were back-extracted with ether and the combined ether layers were dried $\left(\mathrm{MgSO}_{4}\right)$, concentrated and purified by flash chromatography $\left(\mathrm{SiO}_{2}\right.$, hexanes : diethyl ether $=5: 1)$ to afford 2-(2-butynyl)-2-ethoxycarbonylmalonic acid diethyl ester as a yellow oil (4.06 $\mathrm{g}, 91 \%)$.

This oil was dissolved in THF $(20 \mathrm{~mL})$ and added dropwise to a suspension of sodium ethoxide $(1.21 \mathrm{~g}, 17.9 \mathrm{mmol})$ in THF $(20 \mathrm{~mL})$. After stirring at $\mathrm{rt}$ for $2 \mathrm{~h}$ the solution was acidified with $1 \mathrm{~N} \mathrm{HCl}$ and diluted with ether, washed with water, sat. $\mathrm{NaHCO}_{3}$ solution, brine, dried $\left(\mathrm{MgSO}_{4}\right)$, concentrated and purified by flash chromatography $\left(\mathrm{SiO}_{2}\right.$, hexanes : ethyl acetate $\left.=85: 15\right)$ to afford 2-(2-butynyl)malonic acid diethyl ester as a pale yellow oil (2.48 g, 82\%).

A solution of 2-(2-butynyl)malonic acid diethyl ester (0.38 g, $1.8 \mathrm{mmol})$ in THF $(2 \mathrm{~mL})$ was added dropwise to a suspension of $\mathrm{NaH}(72 \mathrm{mg}, 60 \%$ dispersion in mineral oil, $1.8 \mathrm{mmol})$ in THF (6 mL). The resulting clear solution was stirred at $\mathrm{rt}$ for $20 \mathrm{~min}$ and 1-bromo-4-methyl-2,3-pentadiene [prepared by bromination of 4-methyl-2,3pentadien-1-ol (0.20 g, $2.0 \mathrm{mmol})$ with $\mathrm{CBr}_{4}(0.74 \mathrm{~g}, 2.2 \mathrm{mmol})$ and $\mathrm{PPh}_{3}(0.59 \mathrm{~g}, 2.2$ mmol) in $\mathrm{CH}_{2} \mathrm{Cl}_{2}$ at $0 ; \mathrm{C}$ ] was added. The mixture was stirred at $\mathrm{rt}$ overnight and was quenched with sat. $\mathrm{NH}_{4} \mathrm{Cl}$ solution, diluted with ether, washed with brine, sat. $\mathrm{NaHCO}_{3}$ solution, and brine successively, dried $\left(\mathrm{MgSO}_{4}\right)$, concentrated and purified by flash chromatography $\left(\mathrm{SiO}_{2}\right.$, hexanes : diethyl ether $\left.=9: 1\right)$ to afford title compound $\mathbf{8}$ as a colorless oil (0.25 g, 48\%) : ${ }^{1} \mathrm{H}$ NMR (300 MHz, $\left.\mathrm{CDCl}_{3}\right) \delta$ 4.80-4.74 (m, $\left.1 \mathrm{H}\right), 4.24-4.13$ $(\mathrm{m}, 4 \mathrm{H}), 2.78(\mathrm{q}, J=2.4 \mathrm{~Hz}, 2 \mathrm{H}), 2.69(\mathrm{~d}, J=7.6 \mathrm{~Hz}, 2 \mathrm{H}), 1.74(\mathrm{t}, J=2.4 \mathrm{~Hz}, 3 \mathrm{H})$, $1.65(\mathrm{~d}, J=2.8 \mathrm{~Hz}, 6 \mathrm{H}), 1.24(\mathrm{t}, J=7.1 \mathrm{~Hz}, 6 \mathrm{H}) ;{ }^{13} \mathrm{C} \mathrm{NMR}(75 \mathrm{MHz}, \mathrm{CDCl} 3) \delta 203.9$, $170.1,95.0,82.6,78.4,73.5,61.3,57.4,32.5,22.8,20.4,14.0,3.4$; IR (neat) v 1732, 1970, $2981 \mathrm{~cm}^{-1}$; EI-HRMS calcd for $\mathrm{C}_{17} \mathrm{H}_{24} \mathrm{O}_{4}\left[\mathrm{M}^{+}\right] \mathrm{m} / z$. 292.1675, found 292.1688.
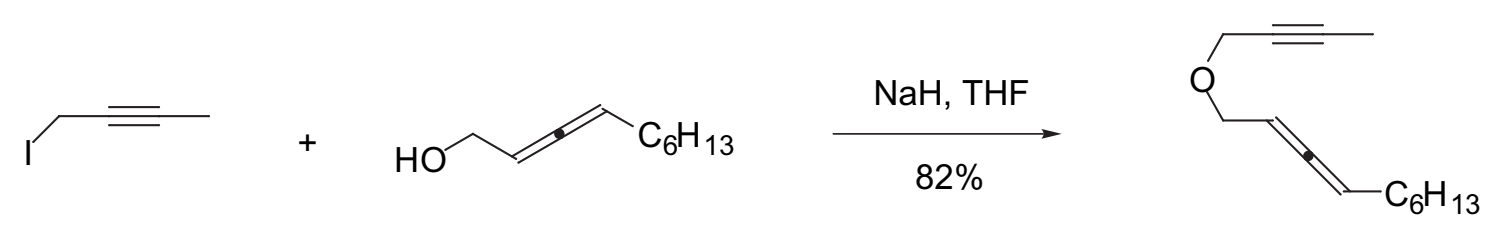
1-(2-Butynyloxy)-2,3-decadiene (25). A solution of 2,3-decadien-1-ol (0.3 g, 1.9 $\mathrm{mmol})$ in THF $(2 \mathrm{~mL})$ was added dropwise to a suspension of $\mathrm{NaH}(0.16 \mathrm{~g}, 60 \%$ dispersion in mineral oil, $3.8 \mathrm{mmol})$ in THF $(10 \mathrm{~mL})$. The resulting clear solution was stirred at $\mathrm{rt}$ for $20 \mathrm{~min}$ and 1-iodo-2-butyne was added. The mixture was stirred at $\mathrm{rt}$ overnight and was quenched with sat. $\mathrm{NH}_{4} \mathrm{Cl}$ solution, diluted with ether, neutralized with $1 \mathrm{~N} \mathrm{HCl}$ solution, washed with brine, sat. $\mathrm{NaHCO}_{3}$ solution, and brine successively, dried $\left(\mathrm{MgSO}_{4}\right)$, concentrated and purified by flash chromatography $\left(\mathrm{SiO}_{2}\right.$, pentane : ethyl ether $=30: 1)$ to afford title compound 25 as a colorless oil $(0.330 \mathrm{~g}, 82 \%):{ }^{1} \mathrm{H}$ NMR $\left(300 \mathrm{MHz}, \mathrm{CDCl}_{3}\right) \delta 5.19-5.13(\mathrm{~m}, 2 \mathrm{H}), 4.09(\mathrm{q}, J=2.2 \mathrm{~Hz}, 2 \mathrm{H}), 4.01(\mathrm{dd}, J=2.9,6.3$ $\mathrm{Hz}, 2 \mathrm{H}), 2.03-1.95$ (m, $2 \mathrm{H}), 1.83(\mathrm{t}, J=2.2 \mathrm{~Hz}, 3 \mathrm{H}), 1.40-1.21(\mathrm{~m}, 8 \mathrm{H}), 0.86(\mathrm{t}, J=6.9$ $\mathrm{Hz}, 3 \mathrm{H}) ;{ }^{13} \mathrm{C}$ NMR $(75 \mathrm{MHz}, \mathrm{CDCl} 3) \delta 205.3,92.0,87.8,82.3,75.0,68.1,57.2,31.6$, 29.0, 28.7, 28.5, 22.6, 14.1, 3.6; IR (neat) $v$ 1963, 2924, $1079 \mathrm{~cm}^{-1}$; EI-HRMS calcd for $\mathrm{C}_{14} \mathrm{H}_{21} \mathrm{O}\left[\mathrm{M}-\mathrm{H}^{+}\right] m / z$. 205.1592, found 205.1589.

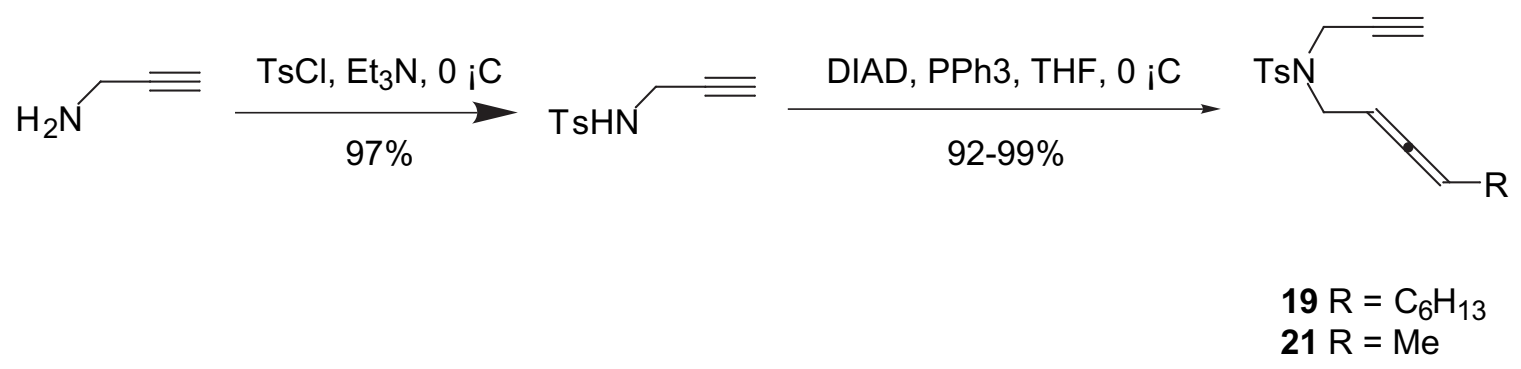

N-(2,3-Decadienyl)-4-methyl-N-(2-propynyl)benzenesulfonamide (19). To a solution of 4-Methyl-N-(2-propynyl)benzenesulfonamide (2.09 g, $10.0 \mathrm{mmol}), \mathrm{PPh}_{3}$ (3.54 g, $13.5 \mathrm{mmol})$ and 2,3-pentadien-1-ol (2.01 g, $13.0 \mathrm{mmol})$ in THF (30 mL) at 0 ¡C was added dropwise diisopropylazodicarboxylate $(2.66 \mathrm{~mL}, 13.5 \mathrm{mmol})$. The resulting solution was allowed to slowly warm to rt and react for overnight. THF was removed in vacuo. The residue was dissolved in ethyl acetate and the resulting solution was washed with brine, dried $\left(\mathrm{Na}_{2} \mathrm{SO}_{4}\right)$, filtered and concentrated in vacuo. The residue was purified by flash chromatography $\left(\mathrm{SiO}_{2}\right.$, hexanes : ethyl acetate $\left.=95: 5\right)$ to afford 19 as a slightly yellow oil (3.42 g, 99\%) : ${ }^{1} \mathrm{H}$ NMR (300 MHz, $\left.\mathrm{CDCl}_{3}\right) \delta 7.70(\mathrm{~d}, J=8.2 \mathrm{~Hz}, 2 \mathrm{H}), 7.26$ $(\mathrm{d}, J=8.2 \mathrm{~Hz}, 2 \mathrm{H}), 5.19-5.13(\mathrm{~m}, 1 \mathrm{H}), 4.99-4.93(\mathrm{~m}, 1 \mathrm{H}), 4.17(\mathrm{dd}, \mathrm{AB}, J=2.4,18.2$ $\mathrm{Hz}, 1 \mathrm{H}), 4.09$ (dd, AB, $J=2.4,18.2 \mathrm{~Hz}, 1 \mathrm{H}), 3.85$ (ddd, A B , J=2.4, 6.9, $14.0 \mathrm{~Hz}, 1$ 
H), 3.76 (ddd, A B , $J=2.4,6.9,14.0 \mathrm{~Hz}, 1 \mathrm{H}), 2.39$ (s, $3 \mathrm{H}), 2.02-1.91$ (m, $3 \mathrm{H}), 1.39$ $1.18(\mathrm{~m}, 8 \mathrm{H}), 0.84(\mathrm{t}, J=7.1 \mathrm{~Hz}, 3 \mathrm{H}) ;{ }^{13} \mathrm{C} \mathrm{NMR}\left(75 \mathrm{MHz}, \mathrm{CDCl}_{3}\right) \delta 205.7,143.5$, 136.1, 129.4, 127.7, 92.8, 85.8, 76.5, 73.5, 46.2, 35.6, 31.6, 28.9, 28.7, 28.5, 22.5, 21.5, 14.0; IR (neat) $v$ 3278, 2926, 1964, 1350, $1163 \mathrm{~cm}^{-1}$; EI-HRMS calcd for $\mathrm{C}_{20} \mathrm{H}_{27} \mathrm{NO}_{2} \mathrm{~S}$ $\left[\mathrm{M}^{+}\right] \mathrm{m} / z$. 345.1763, found 345.1770.

4-Methyl-N-(2,3-pentadienyl)-N-(2-propynyl)benzenesulfonamide (21). To a solution of 4-Methyl-N-(2-propynyl)benzenesulfonamide (0.50 g, $2.4 \mathrm{mmol}), \mathrm{PPh}_{3}(0.85$ g, $3.2 \mathrm{mmol})$ and 2,3-pentadien-1-ol $(0.26 \mathrm{~g}, 3.1 \mathrm{mmol})$ in THF $(10 \mathrm{~mL})$ at 0 ¡C was added dropwise diisopropylazodicarboxylate $(0.64 \mathrm{~mL}, 3.2 \mathrm{mmol})$. The resulting solution was allowed to slowly warm to rt and stirred overnight. THF was removed in vacuo. The residue was dissolved in ethyl acetate and the resulting solution was washed with brine, dried $\left(\mathrm{Na}_{2} \mathrm{SO}_{4}\right)$, filtered and concentrated in vacuo. The residue was purified by flash chromatography $\left(\mathrm{SiO}_{2}\right.$, hexanes : ethyl acetate $\left.=95: 5\right)$ to afford 21 as a colorless solid $(0.61 \mathrm{~g}, 92 \%):{ }^{1} \mathrm{H}$ NMR $\left(300 \mathrm{MHz}, \mathrm{CDCl}_{3}\right) \delta$ 7.75-7.14 (m, $\left.2 \mathrm{H}\right), 7.30-7.26(\mathrm{~m}, 2 \mathrm{H})$, 5.20-5.11 (m, $1 \mathrm{H}), 4.99-4.91(\mathrm{M}, 1 \mathrm{H}), 4.19(\mathrm{dd}, \mathrm{AB}, J=2.5,18.2 \mathrm{~Hz}, 1 \mathrm{H}), 4.12$ (dd, $\mathrm{AB}, J=2.5,18.2 \mathrm{~Hz}, 1 \mathrm{H}), 3.87$ (ddd, A B , $J=2.3,6.8,14.1 \mathrm{~Hz}, 1 \mathrm{H}), 3.79$ (ddd, A B , $J=2.3,6.8,14.1 \mathrm{~Hz}, 1 \mathrm{H}), 2.42(\mathrm{~s}, 3 \mathrm{H}), 2.01(\mathrm{t}, J=2.5 \mathrm{~Hz}, 1 \mathrm{H}), 1.64(\mathrm{dd}, J=3.1,7.1$ $\mathrm{Hz}, 3 \mathrm{H}) ;{ }^{13} \mathrm{C}$ NMR $\left(75 \mathrm{MHz}, \mathrm{CDCl}_{3}\right) \delta 206.6,143.5,136.3,129.5,127.7,87.4,85.3$, 77.4, 73.4, 46.2, 35.7, 21.5, 14.0; IR (neat) v 1966, 1160, 3269, $1597 \mathrm{~cm}^{-1}$; EI-HRMS calcd for $\mathrm{C}_{15} \mathrm{H}_{17} \mathrm{NO}_{2} \mathrm{~S}\left[\mathrm{M}^{+}\right] \mathrm{m} / z$. 275.0980, found 275.0981.

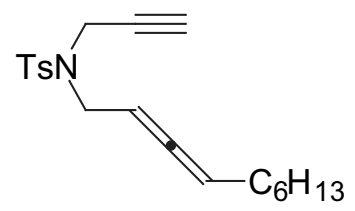

19

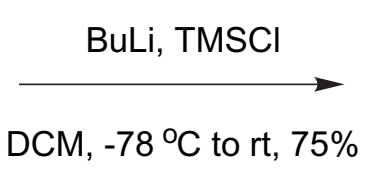

DCM, $-78^{\circ} \mathrm{C}$ to rt, $75 \%$

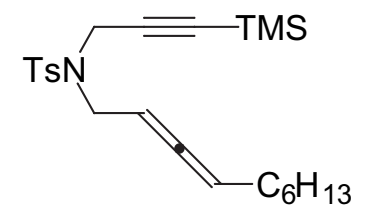

23

$N$-2,3-Decadienyl-4-methyl- $N$-(3-trimethylsilanyl-2-propynyl)benzenesulfon-

amide (23). To a solution of $N$-(2,3-Decadienyl)-4-methyl- $N$-(2-propynyl)benzenesulfonamide $19(0.42 \mathrm{~g}, 1.2 \mathrm{mmol})$ in THF $(10 \mathrm{~mL})$ at ${ }^{-} 78$ i $\mathrm{C}$ was added $1.6 \mathrm{M}$ BuLi solution in hexanes $(1.0 \mathrm{~mL}, 1.6 \mathrm{mmol})$. The mixture was stirred for $30 \mathrm{~min}$ at $-78 ; \mathrm{C}$ 
and treated with trimethylsilyl chloride $(0.32 \mathrm{~mL}, 2.4 \mathrm{mmol})$. The mixture was allowed to gradually warm to rt over 80 minutes and quenched with sat. $\mathrm{NH}_{4} \mathrm{Cl}$ solution, diluted with ether, washed with brine, and dried $\left(\mathrm{MgSO}_{4}\right)$, filtered and concentrated to obtain a yellow oil. Purification by flash chromatography $\left(\mathrm{SiO}_{2}\right.$, hexanes : ethyl acetate $\left.=95: 5\right)$ afforded the product as an off-white solid $(0.38 \mathrm{~g}, 75 \%)$. : ${ }^{1} \mathrm{H} \mathrm{NMR}\left(300 \mathrm{MHz}, \mathrm{CDCl}_{3}\right) \delta$ $7.73(\mathrm{~d}, J=8.3 \mathrm{~Hz}, 2 \mathrm{H}), 7.28(\mathrm{~d}, J=8.3 \mathrm{~Hz}, 2 \mathrm{H}), 5.23-5.16(\mathrm{~m}, 1 \mathrm{H}), 5.03-4.97$ (m, 1 H), $4.21(\mathrm{~d}, \mathrm{AB}, J=18.4 \mathrm{~Hz}, 1 \mathrm{H}), 4.14(\mathrm{~d}, \mathrm{AB}, J=18.4 \mathrm{~Hz}, 1 \mathrm{H}), 3.86(\mathrm{ddd}, \mathrm{A} \mathrm{B}, J=$ 2.3, 6.8, 18.0 Hz, 1 H), 3.78 (ddd, A B , $J=2.3,6.8,18.0 \mathrm{~Hz}, 1 \mathrm{H}), 2.42$ (s, $3 \mathrm{H}), 2.01$ $1.96(\mathrm{~m}, 2 \mathrm{H}), 1.43-1.19(\mathrm{~m}, 8 \mathrm{H}), 0.88(\mathrm{t}, J=6.5 \mathrm{~Hz}, 3 \mathrm{H}), 0.00(\mathrm{~s}, 9 \mathrm{H}) ;{ }^{13} \mathrm{C} \mathrm{NMR}(75$ $\left.\mathrm{MHz}, \mathrm{CDCl}_{3}\right) \delta 205.7,143.3,136.1,129.5,127.7,97.7,92.7,90.7,85.9,46.2,36.6,31.6$, $28.9,28.7,28.5,22.6,21.5,14.1,-0.5$; IR (neat) $v 2925,1964,1351,1164,1094 \mathrm{~cm}^{-1}$; EI-HRMS calcd for $\mathrm{C}_{23} \mathrm{H}_{35} \mathrm{NO}_{2} \mathrm{SSi}\left[\mathrm{M}^{+}\right] \mathrm{m} / z$ 417.2160, found 417.2161.

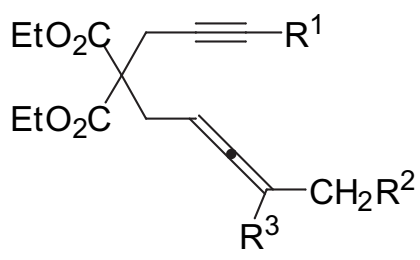

$17 \mathrm{R}^{1}=\mathrm{TMS}, \mathrm{R}^{2}=\mathrm{R}^{3}=\mathrm{H}$

$15 \mathrm{R}^{1}=\mathrm{TMS}, \mathrm{R}^{2}=\mathrm{C}_{5} \mathrm{H}_{13}, \mathrm{R}^{3}=\mathrm{H}$

$13 \mathrm{R}^{1}=\mathrm{R}^{2}=\mathrm{R}^{3}=\mathrm{H}$

$11 \mathrm{R}^{1}=\mathrm{R}^{3}=\mathrm{H}, \mathrm{R}^{2}=\mathrm{C}_{5} \mathrm{H}_{11}$

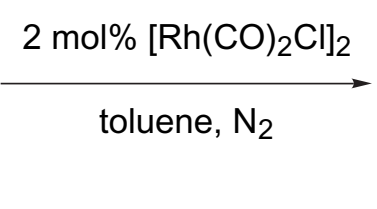

\section{5-Trimethylsilylmethylene-4-vinyl-3-cyclohexene-1,1-dicarboxylic acid}

diethyl ester (18). To a flame-dried test tube equipped with a magnetic stirring bar was added 2-(2,3-pentadienyl)-2-(3-trimethylsilanyl-2-propynyl)malonic acid diethyl ester $(\mathbf{1 7}, 41 \mathrm{mg}, 0.13 \mathrm{mmol})$ and toluene $(0.5 \mathrm{~mL})$. The test tube was evacuated and charged with $\mathrm{N}_{2}$ (this step was repeated 3 times) and $\left[\mathrm{Rh}(\mathrm{CO})_{2} \mathrm{Cl}\right]_{2}\left(2.5 \mathrm{mg}, 6 \times 10^{-3} \mathrm{mmol}\right)$ was 
added. The mixture was stirred at $\mathrm{rt}$ for $1 \mathrm{~h}$ under $\mathrm{N}_{2}$ atmosphere (1 atm). The solvent was removed in vacuo and the residue was purified by flash chromatography $\left(\mathrm{SiO}_{2}\right.$, hexanes : ethyl acetate $=9: 1)$ to afford title compound $\mathbf{1 8}$ as a colorless oil $(18.3 \mathrm{mg}$, 45\%) : ${ }^{1} \mathrm{H}$ NMR (300 MHz, $\left.\mathrm{CDCl}_{3}\right) \delta 6.47(\mathrm{dd}, J=10.8,17.2 \mathrm{~Hz}, 1 \mathrm{H}), 5.94(\mathrm{t}, J=4.3$ $\mathrm{Hz}, 1 \mathrm{H}), 5.64(\mathrm{~s}, 1 \mathrm{H}), 5.32(\mathrm{dd}, J=2.0,17.2 \mathrm{~Hz}, 1 \mathrm{H}), 5.06(\mathrm{dd}, J=2.0,10.8 \mathrm{~Hz}, 1 \mathrm{H})$, 4.27-4.09 (m, $4 \mathrm{H}$ ), 2.97 (s, $2 \mathrm{H}), 2.74$ (d, $J=4.3 \mathrm{~Hz}), 1.23$ (t, $J=7.1 \mathrm{~Hz}), 0.18$ (s, $9 \mathrm{H}$ ); ${ }^{13} \mathrm{C}$ NMR $\left(75 \mathrm{MHz}, \mathrm{CDCl}_{3}\right) \delta 170.8,146.1,138.5,136.0,126.7,124.3,115.4,61.4,54.2$, 35.8, 31.7, 14.0, 0.0; IR (neat) $\vee 1736,1248,1194,1578,2957 \mathrm{~cm}^{-1}$; EI-HRMS calcd for $\mathrm{C}_{18} \mathrm{H}_{28} \mathrm{O}_{4} \mathrm{Si}\left[\mathrm{M}^{+}\right] \mathrm{m} / \mathrm{z} 336.1757$, found 336.1760 .

\section{4-(1-Heptenyl)-5-trimethylsilylmethylene-3-cyclohexene-1,1-dicarboxylic acid}

diethyl ester (16). To a flame-dried test tube equipped with a magnetic stirring bar was added 2-(2,3-decadienyl)-2-(3-trimethylsilyl-2-propynyl)malonic acid diethyl ester (15, $50 \mathrm{mg}, 0.12 \mathrm{mmol})$ and toluene $(0.5 \mathrm{~mL})$. The test tube was evacuated and charged with $\mathrm{N}_{2}$ (this step was repeated 3 times) and $\left[\mathrm{Rh}(\mathrm{CO})_{2} \mathrm{Cl}\right]_{2}\left(2.5 \mathrm{mg}, 6 \times 10^{-3} \mathrm{mmol}\right)$ was added. The mixture was stirred at $\mathrm{rt}$ for $3 \mathrm{~h}$ under $\mathrm{N}_{2}$ atmosphere $(1 \mathrm{~atm})$. The solvent was removed in vacuo and the residue was purified by flash chromatography $\left(\mathrm{SiO}_{2}\right.$, hexanes : ethyl acetate $=9: 1)$ to afford title compound $\mathbf{1 6}$ as a colorless oil $(29.5 \mathrm{mg}, 60 \%, E / Z$ ratio $=3: 1$ determined by ${ }^{1} \mathrm{H}$ NMR). The E/Z isomers were separated by HPLC.

16-E : ${ }^{1} \mathrm{H}$ NMR $\left(300 \mathrm{MHz}, \mathrm{CDCl}_{3}\right) \delta 6.06(\mathrm{~d}, J=15.3 \mathrm{~Hz}, 1 \mathrm{H}), 5.82(\mathrm{t}, J=4.3$ $\mathrm{Hz}, 1 \mathrm{H}), 5.71(\mathrm{td}, J=6.8,15.3 \mathrm{~Hz}, 1 \mathrm{H}), 5.61(\mathrm{~s}, 1 \mathrm{H}), 4.21-4.08(\mathrm{~m}, 4 \mathrm{H}), 2.94(\mathrm{~s}, 2 \mathrm{H})$, 2.69 (d, $J=4.3 \mathrm{~Hz}, 2 \mathrm{H}), 2.06$ (q, $J=6.9 \mathrm{~Hz}, 2 \mathrm{H}), 1.41-1.18(\mathrm{~m}, 6 \mathrm{H}), 1.21$ (t, $J=7.0$ $\mathrm{Hz}, 6 \mathrm{H}), 0.87(\mathrm{t}, J=6.9 \mathrm{~Hz}, 3 \mathrm{H}), 0.14(\mathrm{~s}, 9 \mathrm{H}) ;{ }^{13} \mathrm{C} \mathrm{NMR}\left(75 \mathrm{MHz}, \mathrm{CDCl}_{3}\right) \delta 170.9$, 146.7, 138.0, 132.4, 128.1, 126.3, 123.4, 61.4, 54.2, 35.9, 32.8, 31.7, 31.4, 29.0, 22.5, 14.0, 14.0, 0.1; IR (neat) $v 1736,1247,2926 \mathrm{~cm}^{-1}$; EI-HRMS calcd for $\mathrm{C}_{23} \mathrm{H}_{38} \mathrm{O}_{4} \mathrm{Si}\left[\mathrm{M}^{+}\right]$ $\mathrm{m} / \mathrm{z}$ 406.2539, found 406.2553 .

16-Z : ${ }^{1} \mathrm{H}$ NMR $\left(300 \mathrm{MHz}, \mathrm{CDCl}_{3}\right) \delta 5.91(\mathrm{~d}, J=11.2 \mathrm{~Hz}, 1 \mathrm{H}), 5.66(\mathrm{t}, J=4.0$ Hz, 1 H), 5.61-5.49 (m, 1 H), 5.57 (s, 1 H), 4.24-4.10 (m, 4 H), 2.99 (s, 2 H), 2.74 (d, J= $4.0 \mathrm{~Hz}, 2 \mathrm{H}), 2.02$ (q, $J=6.9 \mathrm{~Hz}, 2 \mathrm{H}), 1.44-1.22(\mathrm{~m}, 6 \mathrm{H}), 1.24(\mathrm{t}, J=7.1 \mathrm{~Hz}, 6 \mathrm{H}), 0.87$ $(\mathrm{t}, J=6.2 \mathrm{~Hz}, 3 \mathrm{H}), 0.16(\mathrm{~s}, 9 \mathrm{H}) ;{ }^{13} \mathrm{C}$ NMR $\left(75 \mathrm{MHz}, \mathrm{CDCl}_{3}\right) \delta 170.9,146.7$, 136.3,133.6, 127.6, 127.1, 125.6, 61.4, 54.3, 35.8, 31.6, 31.5, 29.4, 28.2, 22.5, 14.0, 0.0; 
IR (neat) $v$ 1736, 1247, 1578, 2956, $2856 \mathrm{~cm}^{-1}$; EI-HRMS calcd for $\mathrm{C}_{23} \mathrm{H}_{38} \mathrm{O}_{4} \mathrm{Si}\left[\mathrm{M}^{+}\right] \mathrm{m} / z$ 406.2539, found 406.2547 .

\section{5-Methylene-4-vinyl-cyclohex-3-ene-1,1-dicarboxylic acid diethyl ester(14).}

To a flame-dried test tube equipped with a magnetic stirring bar was added 2-(2,3pentadienyl)-2-(2-propynyl)malonic acid diethyl ester $(13,39 \mathrm{mg}, 0.15 \mathrm{mmol})$ and toluene $(0.5 \mathrm{~mL})$. The test tube was evacuated and charged with $\mathrm{N}_{2}$ (this step was repeated 3 times $)$ then $\left[\mathrm{Rh}(\mathrm{CO})_{2} \mathrm{Cl}\right]_{2}\left(1 \mathrm{mg}, 3 \times 10^{-3} \mathrm{mmol}\right)$ was added. The mixture was stirred and heated at $90\left\lceil\mathrm{C}\right.$ for $1 \mathrm{~h}$ under $\mathrm{N}_{2}$ atmosphere (1 atm). The solvent was removed in vacuo and the residue was purified by flash chromatography $\left(\mathrm{SiO}_{2}\right.$, hexanes : ethyl acetate $=9: 1)$ to afford title compound 14 as a colorless oil $(27.6 \mathrm{mg}, 71 \%):{ }^{1} \mathrm{H}$ NMR $\left(300 \mathrm{MHz}, \mathrm{CDCl}_{3}\right) \delta 6.37(\mathrm{ddd}, J=1.1,10.8,17.3 \mathrm{~Hz}, 1 \mathrm{H}), 5.86(\mathrm{t}, J=4.2 \mathrm{~Hz}, 1$ H), $5.34(\mathrm{dd}, J=1.8,17.3 \mathrm{~Hz}, 1 \mathrm{H}), 5.09$ (s, $1 \mathrm{H}), 5.05$ (dd, $J=1.8,17.3 \mathrm{~Hz}, 1 \mathrm{H}), 4.94$ $(\mathrm{s}, 1 \mathrm{H}), 4.22-4.10(\mathrm{~m}, 4 \mathrm{H}), 2.85(\mathrm{~s}, 2 \mathrm{H}), 2.73(\mathrm{~d}, J=4.2 \mathrm{~Hz}, 2 \mathrm{H}), 1.20(\mathrm{t}, J=7.1 \mathrm{~Hz}, 6$ $\mathrm{H}) ;{ }^{13} \mathrm{C}$ NMR $\left(75 \mathrm{MHz}, \mathrm{CDCl}_{3}\right) \delta 170.8,138.7,136.4,135.0,124.3,115.3,112.4,61.4$, 54.1, 37.4, 31.8, 29.7, 14.1; IR (neat) $v 1734,1244 \mathrm{~cm}^{-1}$; EI-HRMS calcd for $\mathrm{C}_{15} \mathrm{H}_{20} \mathrm{O}_{4}$ $\left[\mathrm{M}^{+}\right] m / z 264.1362$, found 264.1362.

\section{4-(1-Heptenyl)-5-methylene-3-cyclohexene-1,1-dicarboxylic acid diethyl ester}

(12). To a flame-dried test tube equipped with a magnetic stirring bar was added 2-(2,3decadienyl)-2-(2-propynyl)malonic acid diethyl ester (11, $53 \mathrm{mg}, 0.16 \mathrm{mmol})$ and toluene $(0.8 \mathrm{~mL})$. The test tube was evacuated and charged with $\mathrm{N}_{2}$ (this step was repeated 3 times) then $\left[\mathrm{Rh}(\mathrm{CO})_{2} \mathrm{Cl}\right]_{2}\left(1 \mathrm{mg}, 3 \times 10^{-3} \mathrm{mmol}\right)$ was added. The mixture was stirred and heated at $90 ¡ \mathrm{C}$ for $1 \mathrm{~h}$ under $\mathrm{N}_{2}$ atmosphere ( $\left.1 \mathrm{~atm}\right)$. The solvent was removed in vacuo and the residue was purified by flash chromatography $\left(\mathrm{SiO}_{2}\right.$, hexanes : ethyl acetate $=9$ : 1) to afford title compound 12 as a colorless oil $(42.9 \mathrm{mg}, 80 \%, \mathrm{E} / \mathrm{Z}$ ratio $=3: 1$ determined by ${ }^{1} \mathrm{H}$ NMR). The E/Z isomers were separated by HPLC.

12-E : ${ }^{1} \mathrm{H}$ NMR $\left(300 \mathrm{MHz}, \mathrm{CDCl}_{3}\right) \delta 6.00(\mathrm{dd}, J=1.1,15.5 \mathrm{~Hz}, 1 \mathrm{H}), 5.83-5.73$ (m, 2 H), 5.07 (s, $1 \mathrm{H}), 4.90$ (s, $1 \mathrm{H}), 4.19-4.08$ (m, 4 H), 2.84 (s, $2 \mathrm{H}), 2.70(\mathrm{~d}, J=4.1$ Hz, 2 H), 2.04 (q, $J=6.7 \mathrm{~Hz}, 2 \mathrm{H}), 1.41-1.23(\mathrm{~m}, 6 \mathrm{H}), 1.19$ (t, $J=7.1 \mathrm{~Hz}, 6 \mathrm{H}), 0.86$ (t, $J$ $=6.7 \mathrm{~Hz}, 3 \mathrm{H}) ;{ }^{13} \mathrm{C} \mathrm{NMR}\left(75 \mathrm{MHz}, \mathrm{CDCl}_{3}\right) \delta 170.8,139.2,136.0,132.3,127.4,123.0$, 
$112.1,61.3,54.1,37.4,32.8,31.8,31.4,29.7,29.0,22.5,14.0$; IR (neat) v 1736, 1243 , 1181, $2927 \mathrm{~cm}^{-1}$; EI-HRMS calcd for $\mathrm{C}_{20} \mathrm{H}_{30} \mathrm{O}_{4}\left[\mathrm{M}^{+}\right] \mathrm{m} / z$ 334.2144, found 334.2140.

12-Z : ${ }^{1} \mathrm{H}$ NMR $\left(300 \mathrm{MHz}, \mathrm{CDCl}_{3}\right) \delta 5.91(\mathrm{dd}, J=1.4,11.3 \mathrm{~Hz}, 1 \mathrm{H}), 5.61(\mathrm{t}, J=$ $4.8 \mathrm{~Hz}, 1 \mathrm{H}), 5.57$ (td, $J=7.3,11.3 \mathrm{~Hz}, 1 \mathrm{H}), 5.00$ (s, $1 \mathrm{H}), 4.90(\mathrm{~s}, 1 \mathrm{H}), 4.23-4.12$ (m, 4 H), $2.90(\mathrm{~s}, 2 \mathrm{H}), 2.76(\mathrm{~d}, J=2.5 \mathrm{~Hz}, 2 \mathrm{H}), 1.38-1.15(\mathrm{~m}, 6 \mathrm{H}), 1.23(\mathrm{t}, J=7.1 \mathrm{~Hz}, 6 \mathrm{H})$, $0.87(\mathrm{t}, J=6.5 \mathrm{~Hz}, 3 \mathrm{H}) ;{ }^{13} \mathrm{C} \mathrm{NMR}\left(75 \mathrm{MHz}, \mathrm{CDCl}_{3}\right) \delta 1709,139.5,134.5,133.8,126.8$, $125.5,112.5,61.3,54.2,37.1,31.7,31.5,29.5,28.3,22.5,14.0,13.9$; IR (neat) v 1736, 1244, 1182, 1465, $2927 \mathrm{~cm}^{-1}$; EI-HRMS calcd for $\mathrm{C}_{20} \mathrm{H}_{30} \mathrm{O}_{4}\left[\mathrm{M}^{+}\right] \mathrm{m} / z$ 334.2144, found 334.2137 .

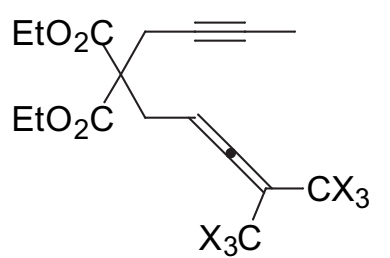

$6 X=D$

$8 \mathrm{X}=\mathrm{H}$

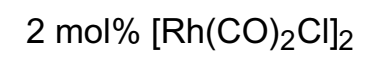

toluene, $90\left\lceil\mathrm{C}, \mathrm{N}_{2}\right.$

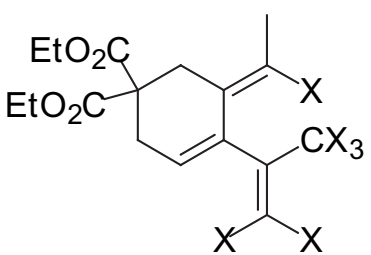

$7 X=D$

$9 \mathrm{X}=\mathrm{H}$

\section{5-Ethylidene-4-isopropenyl-3-cyclohexene-1,1-dicarboxylic acid diethyl ester}

(9). To a flame-dried test tube equipped with a magnetic stirring bar was added 2-(2butynyl)-2-(4-methyl-2,3-pentadienyl)malonic acid diethyl ester (8, $40.0 \mathrm{mg}, 0.14 \mathrm{mmol})$ and toluene $(0.5 \mathrm{~mL})$. The test tube was evacuated and charged with $\mathrm{N}_{2}$ (this step was repeated 3 times $)$ then $\left[\mathrm{Rh}(\mathrm{CO})_{2} \mathrm{Cl}\right]_{2}\left(1 \mathrm{mg}, 3 \times 10^{-3} \mathrm{mmol}\right)$ was added. The mixture was stirred and heated at $90 ; \mathrm{C}$ for $1 \mathrm{~h}$ under $\mathrm{N}_{2}$ atmosphere $(1 \mathrm{~atm})$. The solvent was removed in vacuo and the residue was purified by flash chromatography $\left(\mathrm{SiO}_{2}\right.$, hexanes : ethyl acetate $=9: 1)$ to afford title compound 9 as a colorless oil $(28.0 \mathrm{mg}, 70 \%):{ }^{1} \mathrm{H}$ NMR $\left(300 \mathrm{MHz}, \mathrm{CDCl}_{3}\right) \delta 5.53(\mathrm{q}, J=7.0 \mathrm{~Hz}, 1 \mathrm{H}), 5.45(\mathrm{t}, J=4.1 \mathrm{~Hz}, 1 \mathrm{H}), 4.90-4.89$ $(\mathrm{m}, 1 \mathrm{H}), 4.74(\mathrm{~d}, J=1.9 \mathrm{~Hz}, 1 \mathrm{H}), 4.13(\mathrm{q}, J=7.1 \mathrm{~Hz}, 4 \mathrm{H}), 2.82(\mathrm{~s}, 2 \mathrm{H}), 2.65$ (d, $J=$ $4.1 \mathrm{~Hz}, 2 \mathrm{H}), 1.78(\mathrm{~s}, 3 \mathrm{H}), 1.70(\mathrm{~d}, J=7.0 \mathrm{~Hz}, 3 \mathrm{H}), 1.20(\mathrm{t}, J=7.1 \mathrm{~Hz}, 6 \mathrm{H}) ;{ }^{13} \mathrm{C} \mathrm{NMR}$ $\left(75 \mathrm{MHz}, \mathrm{CDCl}_{3}\right) \delta 171.1,145.2,143.1,130.6,123.4,120.1,114.1,61.3,54.1,31.2$, 30.7, 23.1, 14.0, 13.2; IR (neat) $v 1733,1642,1244 \mathrm{~cm}^{-1}$; EI-HRMS calcd for $\mathrm{C}_{17} \mathrm{H}_{24} \mathrm{O}_{4}$ $\left[\mathrm{M}^{+}\right] m / z 292.1675$, found 292.1679. 


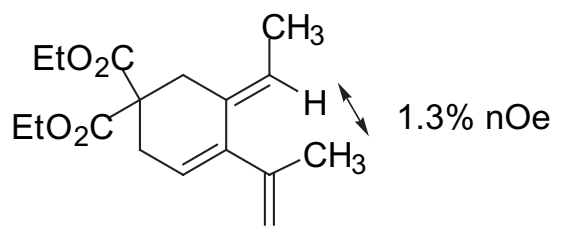

9

\section{5-(1-Deuterioethylidene)-4-(2,2-dideuterio-1-trideuteriomethylvinyl)-3-}

cyclohexene-1,1-dicarboxylic acid diethyl ester (7). To a flame-dried test tube equipped with a magnetic stirring bar was added 2-(2-butynyl)-2-(5,5,5-trideuterio-4trideuteriomethyl-2,3-pentadienyl)malonic acid diethyl ester (6, $40.0 \mathrm{mg}, 0.134 \mathrm{mmol})$ and toluene $(0.5 \mathrm{~mL})$. The test tube was evacuated and charged with $\mathrm{N}_{2}$ (this step was repeated 3 times $)$ then $\left[\mathrm{Rh}(\mathrm{CO})_{2} \mathrm{Cl}\right]_{2}\left(1.0 \mathrm{mg}, 3.0 \times 10^{-3} \mathrm{mmol}\right)$ was added. The mixture was stirred and heated at $90 ; \mathrm{C}$ for $1 \mathrm{~h}$ under $\mathrm{N}_{2}$ atmosphere (1 atm). The solvent was removed in vacuo and the residue was purified by flash chromatography $\left(\mathrm{SiO}_{2}\right.$, hexanes : ethyl acetate $=9: 1)$ to afford title compound 7 as a colorless oil $(25.0 \mathrm{mg}, 63 \%):{ }^{1} \mathrm{H}$ NMR (300 MHz, $\left.\mathrm{CDCl}_{3}\right) \delta 5.45(\mathrm{t}, J=4.1 \mathrm{~Hz}, 1 \mathrm{H}), 4.13(\mathrm{q}, J=7.1 \mathrm{~Hz}, 4 \mathrm{H}), 2.82(\mathrm{~s}, 2$ H), $2.65(\mathrm{~d}, J=4.1 \mathrm{~Hz}, 2 \mathrm{H}), 1.70(\mathrm{~s}, 3 \mathrm{H}), 1.20(\mathrm{t}, J=7.1 \mathrm{~Hz}, 6 \mathrm{H}) ;{ }^{13} \mathrm{C} \mathrm{NMR}(75 \mathrm{MHz}$, $\left.\mathrm{CDCl}_{3}\right) \delta 171.1,144.9,143.0,130.5,120.0,61.3,54.1,31.2,30.7,14.0,13.1$; IR (neat) $v$ 1734, 1244, 1445, $2917 \mathrm{~cm}^{-1}$; EI-HRMS calcd for $\mathrm{C}_{17} \mathrm{H}_{18} \mathrm{D}_{6} \mathrm{O}_{4}\left[\mathrm{M}^{+}\right] \mathrm{m} / z$. 298.2051, found 298.2053.

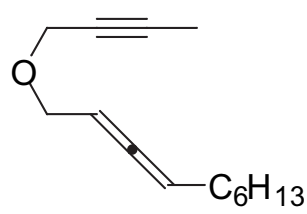

25

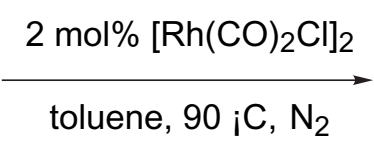

toluene, 90 ¡C, $\mathrm{N}_{2}$

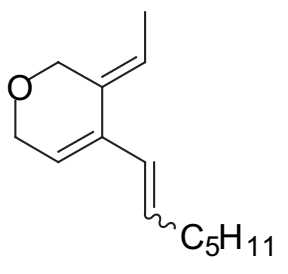

26

3-Ethylidene-4-(1-heptenyl)-3,6-dihydro-2H-pyran (26). To a flame-dried test tube equipped with a magnetic stirring bar was added 1-(2-butynyloxy)-2,3-decadiene $(\mathbf{2 5}, 46.0 \mathrm{mg}, 0.22 \mathrm{mmol})$ and toluene $(0.5 \mathrm{~mL})$. The test tube was evacuated and charged with $\mathrm{N}_{2}$ (this step was repeated 3 times) then $\left[\mathrm{Rh}(\mathrm{CO})_{2} \mathrm{Cl}\right]_{2}(4.6 \mathrm{mg}, 0.01 \mathrm{mmol})$ was added. The mixture was stirred and heated at $90 ; \mathrm{C}$ for $1 \mathrm{~h}$ under $\mathrm{N}_{2}$ atmosphere $(1 \mathrm{~atm})$. The solvent was removed in vacuo and the residue was purified by flash chromatography $\left(\mathrm{SiO}_{2}\right.$, hexanes : ethyl ether $\left.=3: 1\right)$ to afford title compound $\mathbf{2 6}$ as a colorless oil $(25.2$ 
$\mathrm{mg}, 74 \%, \mathrm{E} / \mathrm{Z}$ ratio $=6: 1$ determined by ${ }^{1} \mathrm{H}$ NMR). The E/Z isomers were separated by flash chromatography $\left(\mathrm{SiO}_{2}\right.$, hexanes : ethyl ether $\left.=15: 1\right)$.

26-E : ${ }^{1} \mathrm{H}$ NMR $\left(300 \mathrm{MHz}, \mathrm{CDCl}_{3}\right) \delta 6.05(\mathrm{dd}, J=1.2,15.4 \mathrm{~Hz}, 1 \mathrm{H}), 5.80(\mathrm{td}, J$ $=6.8,15.4 \mathrm{~Hz}, 1 \mathrm{H}), 5.71(\mathrm{t}, J=3.2 \mathrm{~Hz}, 1 \mathrm{H}), 5.61(\mathrm{q}, J=7.1 \mathrm{~Hz}, 1 \mathrm{H}), 4.32(\mathrm{~s}, 2 \mathrm{H})$, $4.24(\mathrm{~d}, J=2.1 \mathrm{~Hz}, 2 \mathrm{H}), 2.07$ (q, $J=6.8 \mathrm{~Hz}, 2 \mathrm{H}), 1.67$ (d, $J=7.1 \mathrm{~Hz}, 3 \mathrm{H}), 1.44-1.17$ $(\mathrm{m}, 6 \mathrm{H}), 0.87(\mathrm{t}, J=6.8 \mathrm{~Hz}, 3 \mathrm{H}) ;{ }^{13} \mathrm{C} \mathrm{NMR}\left(75 \mathrm{MHz}, \mathrm{CDCl}_{3}\right) \delta 134.9,132.9,132.3$, 126.9, 121.3, 119.0, 66.1, 64.4, 32.8, 31.4, 29.7, 29.0, 22.5, 14.0; IR (neat) v 2924, 1132, $1452 \mathrm{~cm}^{-1}$; EI-HRMS calcd for $\mathrm{C}_{14} \mathrm{H}_{22} \mathrm{O}\left[\mathrm{M}^{+}\right] \mathrm{m} / z$ 206.1671, found 206.1671.

26-Z : ${ }^{1} \mathrm{H}$ NMR $\left(300 \mathrm{MHz}, \mathrm{CDCl}_{3}\right) \delta 5.91(\mathrm{ddd}, \mathrm{J}=1.7,3.3,11.4,1 \mathrm{H}), 5.61(\mathrm{td}$, $\mathrm{J}=7.3,11.4,1 \mathrm{H}), 5.57(\mathrm{~s}$, broad, $1 \mathrm{H}), 5.52(\mathrm{dq}, \mathrm{J}=0.7,7.2 \mathrm{~Hz}, 1 \mathrm{H}), 4.38(\mathrm{~s}, 2 \mathrm{H}), 4.29$ (s, broad, 2 H), 2.16-2.08 (m, 2 H), 1.68 (d, J = 7.2 Hz), 1.41-1.25 (m, 6 H), 0.88 (t, J = $6.9 \mathrm{~Hz}, 3 \mathrm{H}$ ); IR (neat) $v$ 2924, 1132, $1451 \mathrm{~cm}^{-1}$; EI-HRMS calcd for $\mathrm{C}_{14} \mathrm{H}_{22} \mathrm{O}\left[\mathrm{M}^{+}\right] \mathrm{m} / \mathrm{z}$ 206.1671, found 206.1670.

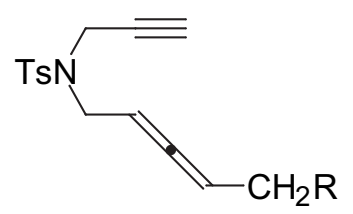

$$
19 \mathrm{R}=\mathrm{C}_{5} \mathrm{H}_{11}
$$

$21 \mathrm{R}=\mathrm{H}$

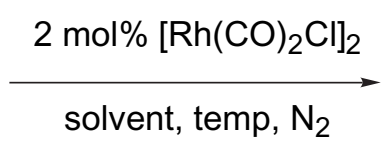

3-Methylene-1-(4-toluenesulfonyl)-4-vinyl-1,2,3,6-tetrahydropyridine (22). To

a dry NMR tube was added 4-methyl-N-(2,3-pentadienyl)-N-(2propynyl)benzenesulfonamide $(\mathbf{2 1}, 26.3 \mathrm{mg}, 0.096 \mathrm{mmol})$ and toluene- $\mathrm{d}_{8}(0.5 \mathrm{~mL})$. The NMR tube was evacuated and charged with $\mathrm{N}_{2}$ (this step was repeated 3 times) then $\left[\mathrm{Rh}(\mathrm{CO})_{2} \mathrm{Cl}\right]_{2}\left(1 \mathrm{mg}, 3 \times 10^{-3} \mathrm{mmol}\right)$ was added. The mixture was allowed to react at $\mathrm{rt}$ for $6 \mathrm{~h}$ until ${ }^{1} \mathrm{H}$ NMR shows complete consumption of starting material. The solvent was removed in vacuo and the residue was purified by flash chromatography $\left(\mathrm{SiO}_{2}\right.$, pentane : ethyl ether $=1: 1)$ to afford title compound 22 as a colorless oil $(24.4 \mathrm{mg}, 93 \%):{ }^{1} \mathrm{H}$ NMR (300 MHz, $\left.\mathrm{CDCl}_{3}\right) \delta 7.67(\mathrm{~d}, J=8.2 \mathrm{~Hz}, 2 \mathrm{H}), 7.30$ (d, $\left.J=8.2 \mathrm{~Hz}, 2 \mathrm{H}\right), 6.31$ (dd, $J=10.8,17.2 \mathrm{~Hz}, 1 \mathrm{H}), 5.76(\mathrm{~s}, 1 \mathrm{H}), 5.28(\mathrm{dd}, J=1.5,17.0 \mathrm{~Hz}, 1 \mathrm{H}), 5.12(\mathrm{~s}, 1 \mathrm{H}), 5.28$ $(\mathrm{dd}, J=1.5,10.8 \mathrm{~Hz}, 1 \mathrm{H}), 5.02(\mathrm{~s}, 1 \mathrm{H}), 3.83-3.81(\mathrm{~m}, 4 \mathrm{H}), 2.42(\mathrm{~s}, 6 \mathrm{H}) ;{ }^{13} \mathrm{C} \mathrm{NMR}(75$ $\left.\mathrm{MHz}, \mathrm{CDCl}_{3}\right) \delta 143.6,136.5,135.4,133.7,133.3,129.5,127.8,121.5,116.6,112.1$, 
49.3, 45.7, 21.5; IR (neat) $v$ 1167, 1347, $1091 \mathrm{~cm}^{-1}$; EI-HRMS calcd for $\mathrm{C}_{15} \mathrm{H}_{17} \mathrm{NO}_{2} \mathrm{~S}$ $\left[\mathrm{M}^{+}\right] m / z 275.0980$, found 275.0985 .

\section{4-(1-Heptenyl)-3-methylene-1-(4-toluenesulfonyl)-1,2,3,6-tetrahydropyridine}

(20). To a dry NMR tube was added N-(2,3-Decadienyl)-4-methyl-N-(2propynyl)benzenesulfonamide $(\mathbf{1 9}, 32.2 \mathrm{mg}, 0.093 \mathrm{mmol})$ and toluene- $\mathrm{d}_{8}(0.5 \mathrm{~mL})$. The NMR tube was evacuated and charged with $\mathrm{N}_{2}$ (this step was repeated 3 times) then $\left[\mathrm{Rh}(\mathrm{CO})_{2} \mathrm{Cl}\right]_{2}(0.7 \mathrm{mg}, 0.002 \mathrm{mmol})$ was added. The mixture was allowed to react at $\mathrm{rt}$ for $6 \mathrm{~h}$ until ${ }^{1} \mathrm{H}$ NMR shows complete consumption of starting material. The solvent was removed in vacuo and the residue was purified by flash chromatography $\left(\mathrm{SiO}_{2}\right.$, hexanes : ethyl acetate $=4: 1)$ to afford title compound 20 as a colorless oil $(24.4 \mathrm{mg}, 90 \%, \mathrm{E} / \mathrm{Z}$ ratio $=6: 1$ measured by ${ }^{1} \mathrm{H}$ NMR). The $E$ and $Z$ isomers were separated by HPLC.

20-E : ${ }^{1} \mathrm{H}$ NMR $\left(300 \mathrm{MHz}, \mathrm{CDCl}_{3}\right) \delta 7.66(\mathrm{~d}, J=8.2 \mathrm{~Hz}, 2 \mathrm{H}), 7.28(\mathrm{~d}, J=8.2$ $\mathrm{Hz}, 2 \mathrm{H}), 5.93(\mathrm{dd}, J=0.9,15.4 \mathrm{~Hz}, 1 \mathrm{H}), 5.70(\mathrm{td}, J=6.8,15.4 \mathrm{~Hz}, 1 \mathrm{H}), 5.66(\mathrm{t}, J=2.7$ Hz, $1 \mathrm{H}), 5.10(\mathrm{~s}, 1 \mathrm{H}), 4.98(\mathrm{~s}, 1 \mathrm{H}), 3.80$ (s, $4 \mathrm{H}), 2.41$ (s, $3 \mathrm{H}), 2.06$ (q, $6.8 \mathrm{~Hz}, 2 \mathrm{H})$, $1.43-1.21(\mathrm{~m}, 6 \mathrm{H}), 0.88(\mathrm{t}, J=6.2 \mathrm{~Hz}, 3 \mathrm{H}) ;{ }^{13} \mathrm{C} \mathrm{NMR}\left(75 \mathrm{MHz}, \mathrm{CDCl}_{3}\right) \delta 143.5,137.1$, $135.1,133.8,133.5,129.5,127.8,126.0,120.3,111.8,49.4,45.8,32.7,31.3,28.8,22.5$, 21.5, 14.0; IR (neat) v 1169, 1354, $2924 \mathrm{~cm}^{-1}$; EI-HRMS calcd for $\mathrm{C}_{20} \mathrm{H}_{27} \mathrm{NO}_{2} \mathrm{~S}\left[\mathrm{M}^{+}\right] \mathrm{m} / z$ 345.1763 , found 345.1754 .

20-Z : ${ }^{1} \mathrm{H}$ NMR $\left(300 \mathrm{MHz}, \mathrm{CDCl}_{3}\right) \delta 7.66(\mathrm{~d}, J=8.2 \mathrm{~Hz}, 2 \mathrm{H}), 7.28(\mathrm{~d}, J=8.2$ Hz, $2 \mathrm{H}), 5.77$ (dd, $J=1.6,11.4 \mathrm{~Hz}, 1 \mathrm{H}), 5.70$ (td, $J=7.2,11.4 \mathrm{~Hz}, 1 \mathrm{H}), 5.51$ (t, $J=2.7$ Hz, $1 \mathrm{H}), 5.01(\mathrm{~s}, 1 \mathrm{H}), 4.94(\mathrm{~s}, 1 \mathrm{H}), 3.84(\mathrm{~s}, 4 \mathrm{H}), 2.41(\mathrm{~s}, 3 \mathrm{H}), 1.92(\mathrm{q}, 6.8 \mathrm{~Hz}, 2 \mathrm{H})$, 1.43-1.20 (m, $6 \mathrm{H}), 0.88(\mathrm{t}, J=7.1 \mathrm{~Hz}, 3 \mathrm{H}) ;{ }^{13} \mathrm{C} \mathrm{NMR}\left(75 \mathrm{MHz}, \mathrm{CDCl}_{3}\right) \delta 143.5,137.4$, $134.7,133.8,133.3,129.6,127.8,125.1,123.0,112.1,49.0,45.5,31.4,29.5,28.4,22.5$, 21.5, 14.0; IR (neat) $v$ 1169, 1351, $2924 \mathrm{~cm}^{-1}$; EI-HRMS calcd for $\mathrm{C}_{20} \mathrm{H}_{27} \mathrm{NO}_{2} \mathrm{~S}\left[\mathrm{M}^{+}\right] \mathrm{m} / z$ 345.1763 , found 345.1748 .

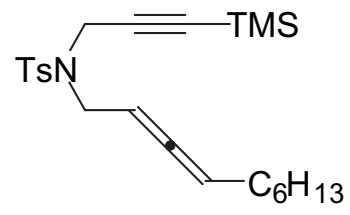

23

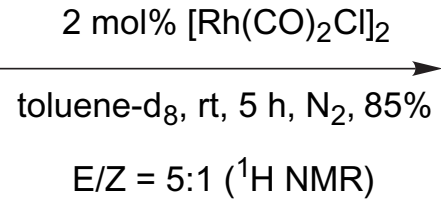

$E / Z=5: 1\left({ }^{1} \mathrm{H} N M R\right)$

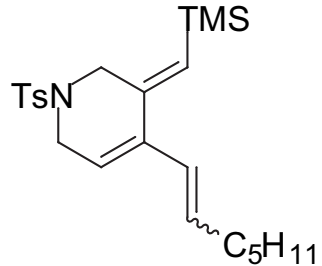

24 


\section{4-(1-Heptenyl)-1-(4-toluenesulfonyl)-3-trimethylsilanylmethylene-1,2,3,6-}

tetrahydropyridine (24). To a dry NMR tube was added $N$-2,3-decadienyl-4-methyl- $N$ (3-trimethylsilanyl-2-propynyl)benzenesulfonamide $(\mathbf{2 3}, 20.6 \mathrm{mg}, 0.049 \mathrm{mmol})$ and toluene- $\mathrm{d}_{8}(0.5 \mathrm{~mL})$. The NMR tube was evacuated and charged with $\mathrm{N}_{2}$ (this step was repeated 3 times $)$ then $\left[\mathrm{Rh}(\mathrm{CO})_{2} \mathrm{Cl}\right]_{2}(0.5 \mathrm{mg}, 0.0012 \mathrm{mmol})$ was added. The mixture was allowed to react at $\mathrm{rt}$ for $5 \mathrm{~h}$ until ${ }^{1} \mathrm{H}$ NMR shows complete consumption of starting material. The solvent was removed in vacuo and the residue was purified by flash chromatography $\left(\mathrm{SiO}_{2}\right.$, hexanes : ethyl acetate $\left.=9: 1\right)$ to afford title compound 24 as a colorless oil $\left(17.5 \mathrm{mg}, 85 \%, \mathrm{E} / \mathrm{Z}\right.$ ratio $=5: 1$ measured by ${ }^{1} \mathrm{H}$ NMR). The $\mathrm{E}$ and $\mathrm{Z}$ isomers were separated by HPLC.

E-24 : ${ }^{1} \mathrm{H}$ NMR (300 MHz, $\left.\mathrm{CDCl}_{3}\right) \delta$ 7.68-7.66 (m, $\left.2 \mathrm{H}\right), 7.32-7.29(\mathrm{~m}, 2 \mathrm{H}), 5.97$ $(\mathrm{dd}, J=1.2,15.3 \mathrm{~Hz}, 1 \mathrm{H}), 5.71-5.59(\mathrm{~m}, 2 \mathrm{H}), 5.66(\mathrm{~s}, 1 \mathrm{H}), 3.90(\mathrm{dd}, \mathrm{AB}, J=0.8,14.0$ Hz, $1 \mathrm{H}), 3.86(\mathrm{dd}, \mathrm{AB}, J=0.8,14.0 \mathrm{~Hz}, 1 \mathrm{H}), 3.79(\operatorname{broad} \mathrm{d}, J=3.5 \mathrm{~Hz}, 2 \mathrm{H}), 2.43(\mathrm{~s}, 3$ H), $2.07(\mathrm{q}, J=6.9 \mathrm{~Hz}, 2 \mathrm{H}), 1.44-1.22(\mathrm{~m}, 6 \mathrm{H}), 0.90(\mathrm{t}, J=6.6 \mathrm{~Hz}, 3 \mathrm{H}), 0.21(\mathrm{~s}, 9 \mathrm{H})$; ${ }^{13} \mathrm{C}$ NMR $\left(75 \mathrm{MHz}, \mathrm{CDCl}_{3}\right) \delta 144.6,143.4,137.3,134.2,133.8,129.5,127.9,126.9$, $126.8,120.5,48.2,45.6,32.7,31.3,28.9,22.5,21.4,14.0,0.01$; IR (neat) $v 2955,2855$, 1584, 1352, $1167 \mathrm{~cm}^{-1}$; EI-HRMS calcd for $\mathrm{C}_{23} \mathrm{H}_{35} \mathrm{NO}_{2} \mathrm{SSi}\left[\mathrm{M}^{+}\right] \mathrm{m} / z$ 417.2160, found 417.2160.

Z-24 : ${ }^{1} \mathrm{H}$ NMR (300 MHz, $\left.\mathrm{CDCl}_{3}\right) \delta$ 7.70-7.67 (m, $\left.2 \mathrm{H}\right), 7.32-7.29$ (m, $\left.2 \mathrm{H}\right), 5.78$ (dd, $J=1.4,11.4 \mathrm{~Hz}, 1 \mathrm{H}), 5.63-5.52$ (m, $2 \mathrm{H}), 5.59$ (s, $1 \mathrm{H}), 3.93$ (broad s, $2 \mathrm{H}), 3.84-$ $3.82(\mathrm{~m}, 2 \mathrm{H}), 2.43(\mathrm{~s}, 3 \mathrm{H}), 1.92(\mathrm{q}, 6.9 \mathrm{~Hz}, 2 \mathrm{H}), 1.35-1.21(\mathrm{~m}, 6 \mathrm{H}), 0.88(\mathrm{t}, J=6.7 \mathrm{~Hz}$, $3 \mathrm{H}), 0.21$ (s, $9 \mathrm{H})$; IR (neat) v 2955, 2926, 2856, 1597, 1352, $1168 \mathrm{~cm}^{-1}$; EI-HRMS calcd for $\mathrm{C}_{23} \mathrm{H}_{35} \mathrm{NO}_{2} \mathrm{SSi}\left[\mathrm{M}^{+}\right] m / z$ 417.2160, found 417.2164. 


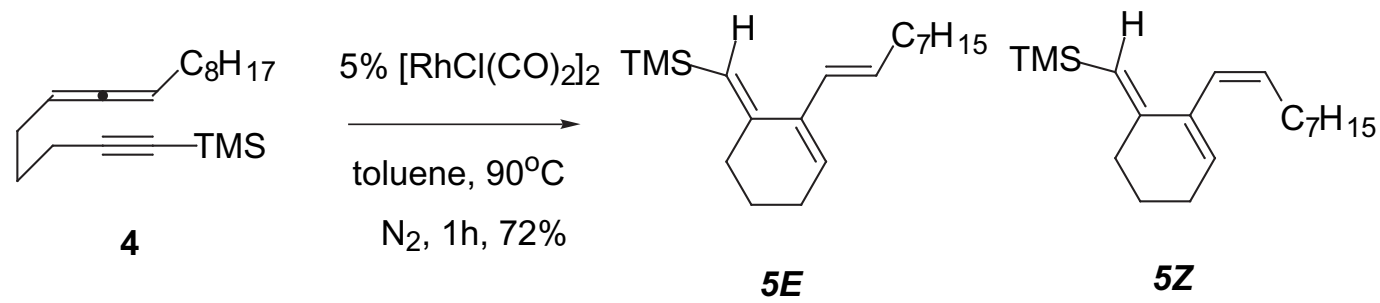

Procedure for $\left[\mathrm{RhCl}(\mathrm{CO})_{2}\right]_{2}$ Catalyzed Triene Formation. A flame dried 2-neck flask was charged with $\left[\mathrm{RhCl}(\mathrm{CO})_{2}\right]_{2}\left(3.5 \mathrm{mg}, 9.0 \times 10^{-3} \mathrm{mmol}\right)$ and flushed with a stream of nitrogen for $5 \mathrm{~min}$. Allenyne $4(56 \mathrm{mg}, 0.10 \mathrm{mmol})$ in $5 \mathrm{~mL}$ of dry toluene was then added via syringe and the reaction mixture was immersed in a preheated $90{ }^{\mathrm{i}} \mathrm{C}$ sand bath. After $1 \mathrm{~h}$ TLC showed complete loss of starting material and the reaction was cooled to $\mathrm{rt}$ and concentrated under reduced pressure. Trienes $\mathbf{5 E - Z}$ were purified by flash silica gel chromatography eluting with hexanes to afford $40 \mathrm{mg}(0.14 \mathrm{mmol})$ of the triene in $72 \%$ yield as a $5: 1$ mixture of $E: Z$ isomers. Trimethyl-(2-oct-1-enyl-cyclohex-2enylidenemethyl)-silane. 5E. ${ }^{1} \mathrm{H}$ NMR $\left(300 \mathrm{MHz}, \mathrm{CDCl}_{3}\right) \delta: 6.13(\mathrm{dd}, 1 \mathrm{H}, J=15.4$ $\mathrm{Hz}), 5.92(\mathrm{t}, 1 \mathrm{H}, J=4.2 \mathrm{~Hz}), 5.77(\mathrm{dt}, 1 \mathrm{H}, J=15.4,6.8 \mathrm{~Hz}), 5.52(\mathrm{~s}, 1 \mathrm{H}), 2.46-2.42(\mathrm{~m}$, 2H), $2.22(\mathrm{q}, 2 \mathrm{H}, J=5.4 \mathrm{~Hz}), 2.11(\mathrm{q}, 2 \mathrm{H}, J=6.9 \mathrm{~Hz}), 1.74(\mathrm{p}, 2 \mathrm{H}, J=6.2 \mathrm{~Hz}), 1.43-$ $1.28(\mathrm{~m}, 12 \mathrm{H}), 0.90(\mathrm{t}, 3 \mathrm{H}, J=6.9 \mathrm{~Hz}), 0.15(\mathrm{~s}, 9 \mathrm{H}) ;{ }^{13} \mathrm{C} \mathrm{NMR}\left(300 \mathrm{MHz}, \mathrm{CDCl}_{3}\right) \delta$ : $152.4,138.7,131.8,129.5,127.5,123.1,33.5,32.1,31.9,29.9,29.7,29.5,29.4,26.6$, 23.7, 22.9, 0.4. IR (neat) 2954, 2927, 2855, 1248, $841 \mathrm{~cm}^{-1}$.

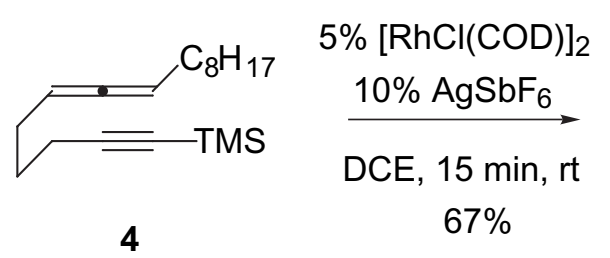

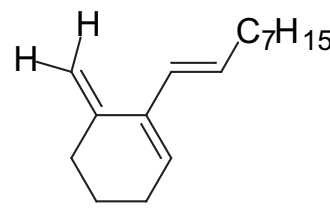

Desilyated 5E

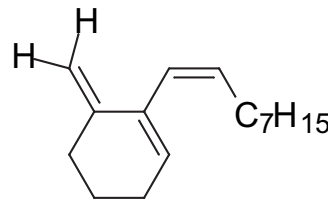

Desilylated $5 Z$

Procedure for $[\mathbf{R h C l}(\mathbf{C O D})]_{2}$ Catalyzed Triene Formation. Allenyne 4 (47 mg, 0.17 mmol) was azeotroped with benzene $(3 \times 1 \mathrm{~mL})$ and placed on a high-vac manifold for 20 min. Allenyne 4 was then diluted with $3 \mathrm{~mL}$ of dry 1,2-dichloroethane and the solution was degassed with a stream of $\mathrm{N}_{2}$ for 5 min. To the reaction mixture was then added 
$[\mathrm{RhCl}(\mathrm{COD})]_{2}\left(4.5 \mathrm{mg}, 9.1 \times 10^{-3} \mathrm{mmol}\right)$ and the reaction stirred at $\mathrm{rt}$ for $5 \mathrm{~min}$ during which time the reaction mixture turned a bright yellow color. To the reaction mixture was then added $\mathrm{AgSbF}_{6}\left(5.9 \mathrm{mg}, 1.7 \times 10^{-2} \mathrm{mmol}\right)$ after which the reaction turned a deep red color. After $15 \mathrm{~min}$ at $\mathrm{rt}$ TLC showed complete loss of starting material and the reaction mixture was diluted with $5 \mathrm{~mL}$ of $\mathrm{Et}_{2} \mathrm{O}$ and filtered through a plug of celite $545^{\circledR}$. The filtrate was concentrated under reduced pressure and the products purified by silica gel chromatography eluting with hexanes to afford $23 \mathrm{mg}(0.12 \mathrm{mmol})$ of desilylated triene 5 as a $13: 1$ mixture of $E: Z$ isomers in 67\% yield. 6-Methylene-1-oct-1-enylcyclohexene. 5-E ${ }^{1} \mathrm{H}$ NMR $\left(300 \mathrm{MHz}, \mathrm{CDCl}_{3}\right) \delta: 6.10(\mathrm{~d}, 1 \mathrm{H}, J=15.4 \mathrm{~Hz}), 5.88(\mathrm{t}, 1 \mathrm{H}$, $J=3.8 \mathrm{~Hz}), 5.81(\mathrm{dt}, 1 \mathrm{H}, J=15.4,6.8 \mathrm{~Hz}), 4.97$ (s, 1H), $4.79(\mathrm{~s}, 1 \mathrm{H}), 2.35$ (t, 2H, $J=6.1$ $\mathrm{Hz}), 2.21$ (q, 2H, $J=5.5 \mathrm{~Hz}), 2.09$ (q, 2H, $J=6.9 \mathrm{~Hz}), 1.71$ (p, 2H, $J=6.15 \mathrm{~Hz}), 1.43-$ $1.19(\mathrm{~m}, 8 \mathrm{H}), 0.89(\mathrm{t}, 3 \mathrm{H}, J=6.5 \mathrm{~Hz}) ;{ }^{13} \mathrm{C} \mathrm{NMR}\left(300 \mathrm{MHz}, \mathrm{CDCl}_{3}\right) \delta: 144.0,136.9$, 131.6, 128.8, 127.2, 109.0, 33.2, 32.8, 29.7, 29.1, 26.7, 23.6, 22.9, 14.3; IR (neat) 2930, 2856, 1456 $\mathrm{cm}^{-1}$; MS (GC/MS) m/e $204(\mathrm{M}+)$, 147, 133, 119, 105, 91, 84; HRMS calcd for $\mathrm{C}_{15} \mathrm{H}_{24}: 204.1878$; found : 204.1880. 5- $\boldsymbol{Z}:{ }^{1} \mathrm{H}$ NMR (300 MHz, $\left.\mathrm{CDCl}_{3}\right) \delta 5.96(\mathrm{~d}$, 2H, $J=11.3 \mathrm{~Hz}$ ), 5.70 (br s, 1H), 5.55 (dt, 1H, $J=11.3,7.2 \mathrm{~Hz}), 4.87$ (s, 1H), 4.75 (s, $1 \mathrm{H}), 2.41-2.36(\mathrm{~m}, 2 \mathrm{H}), 2.23$ (q, 2H, $J=5.1 \mathrm{~Hz}), 2.10$ (q, 2H, $J=7.1 \mathrm{~Hz}), 1.74$ (p, 2H, $J$ $=6.2 \mathrm{~Hz}), 1.37-1.27(\mathrm{~m}, 8 \mathrm{H}), 0.89(\mathrm{t}, 3 \mathrm{H}, J=6.7 \mathrm{~Hz}) ;{ }^{13} \mathrm{C} \mathrm{NMR}\left(75 \mathrm{MHz}, \mathrm{CDCl}_{3}\right) \delta$ $143.8,134.4,132.6,129.4,127.8,109.1,32.1,31.7,29.9,28.9,28.4,26.2,23.2,22.6$, 14.1; MS (GC/MS) m/e $204(\mathrm{M}+), 147,133,119,105,91,79$; IR (neat) 2925, 2854, $1455 \mathrm{~cm}^{-1}$.

Procedure for $\left[\mathrm{RhCl}\left(\mathrm{CH}_{2} \mathrm{CH}_{2}\right)\right]_{2}$ Catalyzed Triene Formation. Allenyne 4 (45 $\mathrm{mg}$, $0.16 \mathrm{mmol})$ was azeotroped with benzene $(3 \times 1 \mathrm{~mL})$ and placed on a high-vac manifold for $20 \mathrm{~min}$ The allenyne 4 was then diluted with $3 \mathrm{~mL}$ of dry 1,2-dichloroethane and the solution degassed with a stream of $\mathrm{N}_{2}$ for $5 \mathrm{~min}$ To the reaction mixture was then added $\left[\mathrm{RhCl}\left(\mathrm{CH}_{2} \mathrm{CH}_{2}\right)_{2}\right]_{2}\left(3.0 \mathrm{mg}, 7.7 \times 10^{-3} \mathrm{mmol}\right)$ and stirred at $\mathrm{rt}$ for $5 \mathrm{~min}$ during which time the reaction turned to light gold. To the reaction mixture was then added $\mathrm{AgSbF}_{6}$ (6.0 mg, $\left.1.7 \times 10^{-2} \mathrm{mmol}\right)$ after which time the reaction mixture turned dark red. After 15 min at rt TLC showed complete loss of starting material and the reaction was diluted with $5 \mathrm{~mL}$ of $\mathrm{Et}_{2} \mathrm{O}$ and filtered through a plug of Celite $545^{\circledR}$. The filtrate was concentrated 
under reduced pressure and the products purified by silica gel chromatography eluting with hexanes to afford $15.3 \mathrm{mg}\left(7.5 \times 10^{-2} \mathrm{mmol}\right)$ of desilylated triene 5 as a $4: 1$ mixture of $E: Z$ isomers in $46 \%$ yield.

\section{General procedure for performing the Allenic Alder-ene reaction catalyzed}

by an Ir complex. To a flame-dried test tube was added the substrate ( 1 eq), dichloroethane (DCE, $0.50 \mathrm{~mL} / 1 \mathrm{mmol}$ of substrate). The test tube was evacuated and charged with $\mathrm{N}_{2}$ (this step was repeated 3 times). A solution of $\mathrm{AgBF}_{4}$ in $\mathrm{DCE}(0.05 \mathrm{M}$, 0.2 eq) was added to the test tube followed by immediate addition of $[\operatorname{Ir}(\mathrm{COD}) \mathrm{Cl}]_{2}(0.1$ eq). The mixture was heated at $60 ¡ \mathrm{C}$ for $5 \mathrm{~min}$, cooled to $0 \nmid \mathrm{C}$, diluted with hexanes and passed through a short $\mathrm{SiO}_{2}$ column to remove the catalyst. Purification of the crude products by flash chromatography $\left(\mathrm{SiO}_{2}\right.$, hexanes : ethyl acetate $\left.=9: 1\right)$ afforded the desired products and the $E / Z$ ratios were measured by ${ }^{1} \mathrm{H}$ NMR or by GC analysis.

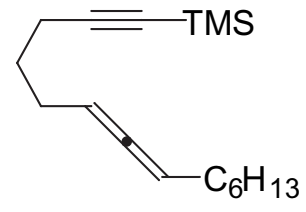

4

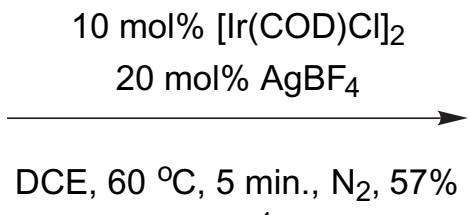

$\mathrm{E} / \mathrm{Z}>20: 1\left({ }^{1} \mathrm{H}\right.$ NMR)

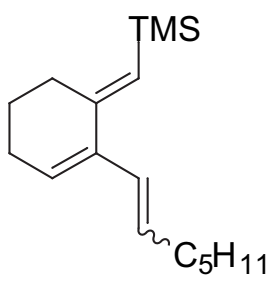

5

Allenyne 4 (28 mg, $0.10 \mathrm{mmol})$ was treated under the general iridium conditions described above to afford triene $5(16 \mathrm{mg}, 57 \%)$ in an $E / Z$ ratio of more than $20: 1$ (determined by ${ }^{1} \mathrm{H}$ NMR analysis). 


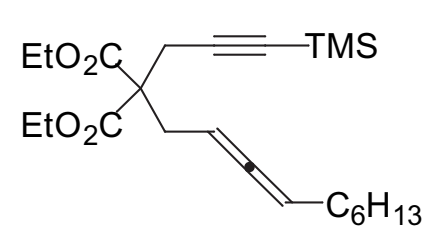

15

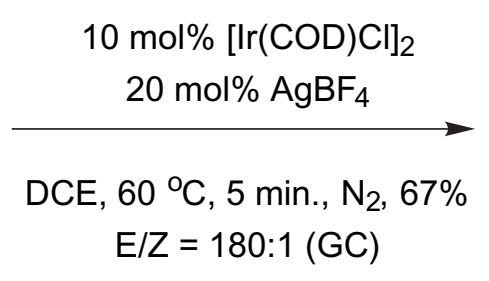

$E / Z=180: 1(G C)$

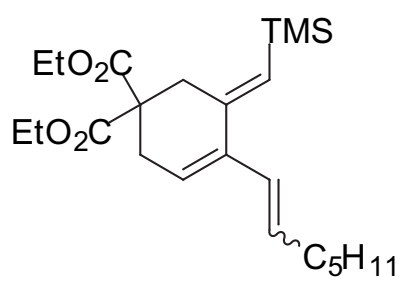

16

Allenyne 15 (43 mg, $0.11 \mathrm{mmol})$ was treated to the general iridium conditions described above to afford triene $16(29 \mathrm{mg}, 67 \%)$ in an $E / Z$ ratio of $180: 1$ (determined by GC analysis).

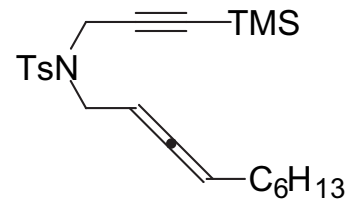

23

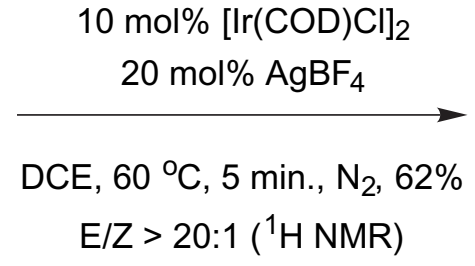

$E / Z>20: 1\left({ }^{1} H\right.$ NMR)

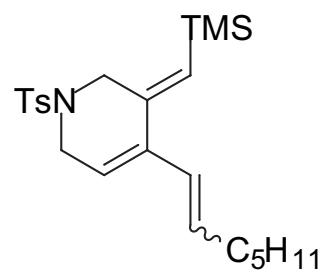

24

Allenyne 23 (40 mg, $0.096 \mathrm{mmol}$ ) was treated to the general iridium conditions described above to afford triene $24(25 \mathrm{mg}, 62 \%)$ in an $E / Z$ ratio of more than $20: 1$ (determined by ${ }^{1} \mathrm{H}$ NMR analysis).<smiles>C#CCCCC(=O)O</smiles>

$\mathrm{H}$
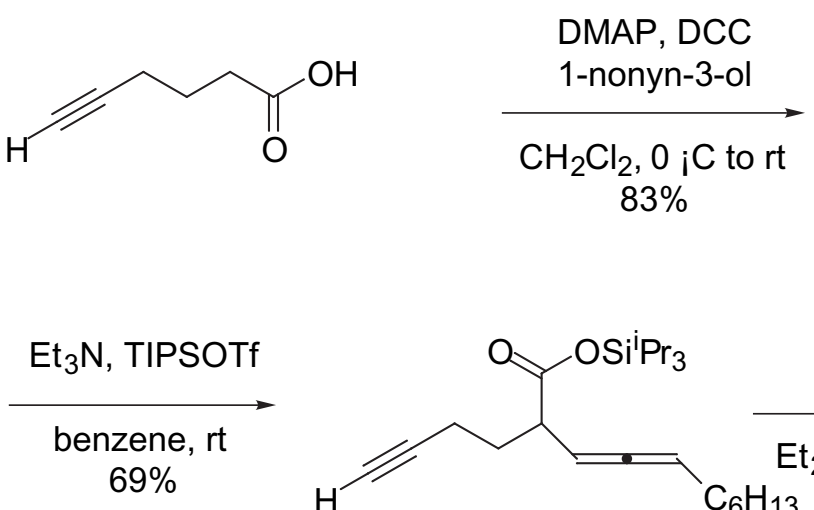

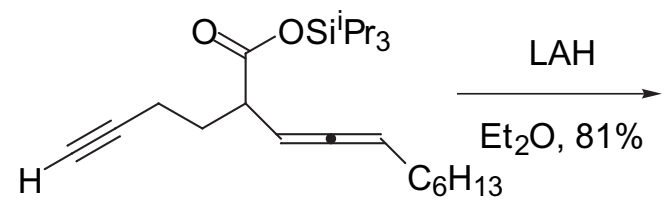

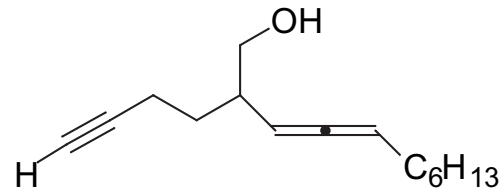

27 
2-(3-Butynyl)-3,4-undecadien-1-ol (27). To a stirred solution of 5-hexyn-1carboxylic acid (1.52 g, $13.6 \mathrm{mmol})$ in dichloromethane $(13 \mathrm{~mL})$ were added 4dimethylaminopyridine (70 mg, $0.57 \mathrm{mmol})$ and 1-nonyn-3-ol $(1.90 \mathrm{~g}, 13.6 \mathrm{mmol})$. The solution was cooled to $0{ }^{\circ} \mathrm{C}$ and 1,3-dicyclohexylcarbodiimide $(2.81 \mathrm{~g}, 13.6 \mathrm{mmol})$ was added. The reaction was stirred at $0{ }^{\circ} \mathrm{C}$ for 5 min then warmed to $\mathrm{rt}$ and stirred for an additional $1 \mathrm{~h}$. The precipitate was filtered off and the resulting filtrate was concentrated in vacuo. The crude material was purified by flash chromatography on silica gel column using 5\% ethyl acetate/hexanes as the eluant to afford hex-5-ynoic acid 1-hexyl-prop-2ynyl ester as a yellow oil (2.62 g, 83\%).

To a stirred solution of 5-hexynoic acid 1-hexyl-prop-2-ynyl ester (1.20 g, 5.13 mmol) in benzene $(4 \mathrm{~mL})$ were added triethylamine $(1.44 \mathrm{~mL}, 10.26 \mathrm{mmol})$ and triisopropylsilyl trifluoromethanesulfonate $(2.1 \mathrm{~mL}, 7.7 \mathrm{mmol})$. The solution was stirred at $\mathrm{rt}$ for $9 \mathrm{~h}$. The two layers were separated and the bottom layer was extracted with benzene. The benzene layers were combined and concentrated. The crude material was purified by flash column chromatography on silica gel using $5 \%$ ethyl acetate/hexanes as the eluant to give 2-but-3-ynyl-undeca-3,4-dienoic acid methyl ester (1.38 g, $3.54 \mathrm{mmol})$ as a colorless oil.

This oil (1.38 g, $3.54 \mathrm{mmol})$ was dissolved in ether $(7 \mathrm{~mL})$ and the solution was cooled to $0{ }^{\circ} \mathrm{C}$. To this solution was added lithium aluminum hydride $(115 \mathrm{mg}, 3.03$ $\mathrm{mmol})$. The reaction was stirred at $0{ }^{\circ} \mathrm{C}$ for $2 \mathrm{~h}$. Water $(115 \mu \mathrm{L}), 15 \% \mathrm{NaOH}(115 \mu \mathrm{L})$ and water $(345 \mu \mathrm{L})$ were added successively. The resulting white precipitate was filtered off and the filtrate was concentrated. The crude material was purified by flash chromatography using $15 \%$ ethyl acetate/hexanes as the eluant to afford title compound 27 as a colorless oil $(631 \mathrm{mg}, 81 \%):{ }^{1} \mathrm{H}$ NMR $\left(300 \mathrm{MHz}, \mathrm{CDCl}_{3}\right) \delta 5.20$ (dq, $J=2.0,6.7$ Hz, 1 H), 5.01-4.95 (m, 1 H), 3.64-3.51 (m, 2 H), 2.42-2.19 (m, 3 H), 2.05-1.95 (m, 3 H), 1.79-1.67 (m, $1 \mathrm{H}), 1.61-1.51(\mathrm{~m}, 2 \mathrm{H}), 1.44-1.29(\mathrm{~m}, 8 \mathrm{H}), 0.89(\mathrm{t}, J=7.0 \mathrm{~Hz}, 3 \mathrm{H}) ;{ }^{13} \mathrm{C}$ NMR (75 MHz, $\left.\mathrm{CDCl}_{3}\right) \delta 204.3,92.4,91.0,84.1,68.5,65.7,41.2,31.6,29.8,29.1,28.9$, 28.8, 22.6, 16.2, 14.0; IR (film) 3365, 3309, 2930, 2858, 2121, 1958, 1460, $1035 \mathrm{~cm}^{-1}$. 


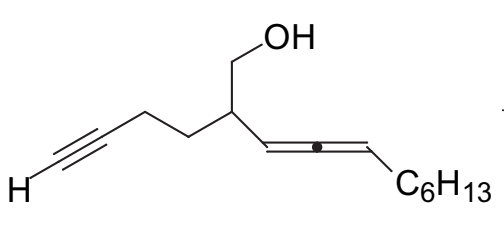

27

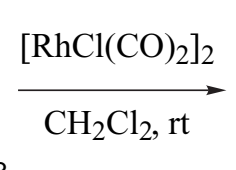

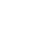
-

3-(1-Heptenyl)-4-methylene-2-cyclohexenylmethanol (28). To a stirred solution of 2-(3-Butynyl)-3,4-undecadien-1-ol (27, $50 \mathrm{mg}, 0.23 \mathrm{mmol})$ in $2 \mathrm{~mL}$ of $\mathrm{CH}_{2} \mathrm{Cl}_{2}$ was added chlorodicarbonylrhodium (I) dimer $(4 \mathrm{mg}, 0.01 \mathrm{mmol})$. The reaction was stirred at $\mathrm{rt}$ for $6 \mathrm{~h}$. After removal of $\mathrm{CH}_{2} \mathrm{Cl}_{2}$, the resulting crude material was purified by flash chromatography on silica gel using 5\% ethyl acetate/hexanes as the eluant to afford 28-E (27 mg, 54\%) and 28-Z (13 mg, 26\%) as colorless oil.

28-E : ${ }^{1} \mathrm{H}$ NMR $\left(300 \mathrm{MHz}, \mathrm{CDCl}_{3}\right) \delta 6.11(\mathrm{dt}, J=1.2,15.4 \mathrm{~Hz}, 1 \mathrm{H}), 5.86(\mathrm{dt}, J=$ 6.8, $15.4 \mathrm{~Hz}, 1 \mathrm{H}), 5.80(\mathrm{~s}, 1 \mathrm{H}), 5.03(\mathrm{~s}, 1 \mathrm{H}), 4.86(\mathrm{~s}, 1 \mathrm{H}), 3.60(\mathrm{t}, J=5.7 \mathrm{~Hz}, 1 \mathrm{H})$, 2.58-2.43 (m, $2 \mathrm{H}), 2.38-2.29$ (m, $1 \mathrm{H}), 2.10$ (q, $J=6.8 \mathrm{~Hz}, 2 \mathrm{H}), 1.94-1.85(\mathrm{~m}, 1 \mathrm{H})$, 1.55-1.40 (m, $4 \mathrm{H}), 1.37-1.26(\mathrm{~m}, 4 \mathrm{H}), 0.90(\mathrm{t}, J=6.8 \mathrm{~Hz}, 3 \mathrm{H}) ;{ }^{13} \mathrm{C}$ NMR $(75 \mathrm{MHz}$, $\left.\mathrm{CDCl}_{3}\right) \delta 143.4,138.0,132.2,128.2,126.9,109.6,66.8,39.7,32.9,31.4,31.0,29.0,26.2$, 22.5, 14.0; IR (film) 3329, 2925, 2853, 1629, 1465, $1045 \mathrm{~cm}^{-1}$; EI-HRMS calcd for $\mathrm{C}_{15} \mathrm{H}_{24} \mathrm{O}\left[\mathrm{M}^{+}\right] \mathrm{m} / \mathrm{z} 220.1827$, found 220.1832).

28-Z : ${ }^{1} \mathrm{H}$ NMR (300 MHz, $\left.\mathrm{CDCl}_{3}\right) \delta 5.98(\mathrm{~d}, J=11.4 \mathrm{~Hz}, 1 \mathrm{H}), 5.63-5.54(\mathrm{~m}, 2$ H), 4.92 (s, $1 \mathrm{H}), 4.81$ (s, $1 \mathrm{H}), 3.62(\mathrm{t}, J=4.8 \mathrm{~Hz}, 2 \mathrm{H}), 2.60-2.46$ (m, $2 \mathrm{H}), 2.41-2.30$ (m, $1 \mathrm{H}), 2.10(\mathrm{qd}, J=1.3,6.9 \mathrm{~Hz}, 2 \mathrm{H}), 1.97-1.87(\mathrm{~m}, 1 \mathrm{H}), 1.58-1.48(\mathrm{~m}, 1 \mathrm{H}), 1.41-$ 1.27 (m, $7 \mathrm{H}), 0.88(\mathrm{t}, J=6.8 \mathrm{~Hz}, 3 \mathrm{H}) ;{ }^{13} \mathrm{C} \mathrm{NMR}\left(75 \mathrm{MHz}, \mathrm{CDCl}_{3}\right) \delta 143.4,136.0$, 133.1, 129.5, 127.5, 110.1, 69.7, 66.8, 39.5, 31.5, 30.4, 29.6, 28.4, 26.0, 22.5, 14.1; IR (film) $3329,2925,2853,1465,1045 \mathrm{~cm}^{-1}$; EI-HRMS calcd for $\mathrm{C}_{15} \mathrm{H}_{24} \mathrm{O}\left[\mathrm{M}^{+}\right] \mathrm{m} / \mathrm{z}$ 220.1827 , found 220.1832 . 

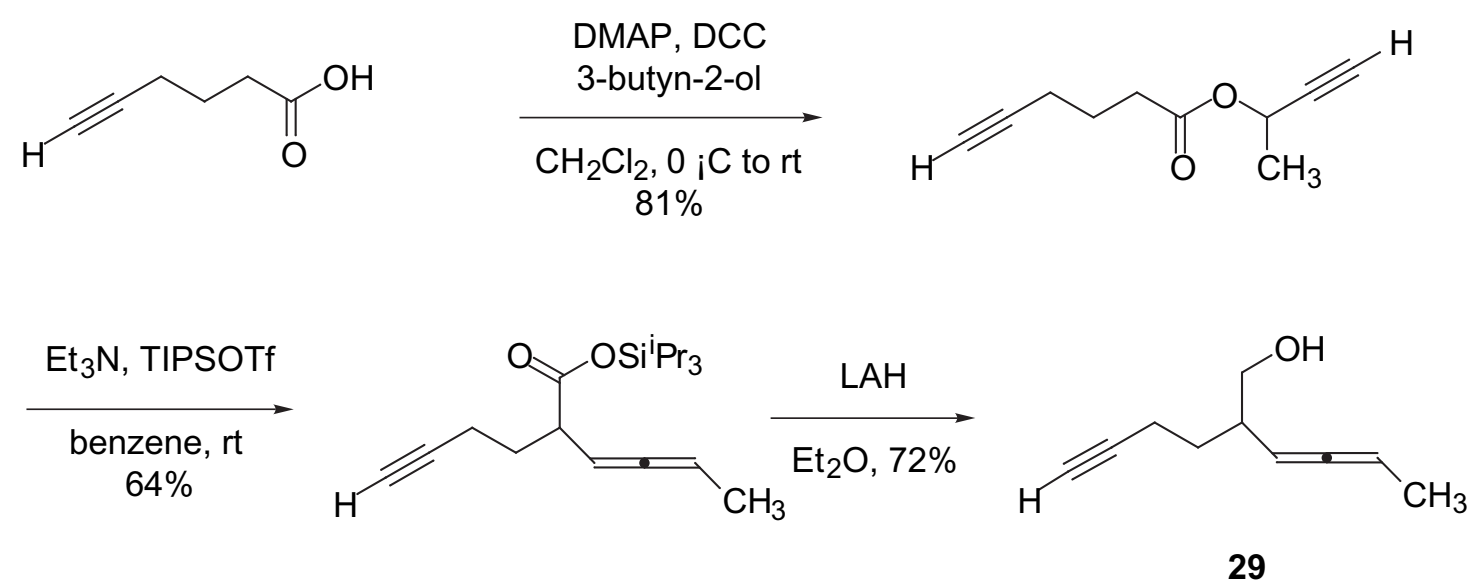

2-(3-Butynyl)-3,4-hexadien-1-ol (29). To a stirred solution of 5-hexyn-1carboxylic acid $(1.49 \mathrm{~g}, 13.3 \mathrm{mmol})$ in dichloromethane $(13 \mathrm{~mL})$ were added 4dimethylaminopyridine (163 mg, $1.33 \mathrm{mmol})$ and 3-butyn-2-ol (1.12 g, $16.0 \mathrm{mmol})$. The solution was cooled to $0{ }^{\circ} \mathrm{C}$ and 1,3-dicyclohexylcarbodiimide $(3.30 \mathrm{~g}, 16.0 \mathrm{mmol})$ was added. The reaction was stirred at $0{ }^{\circ} \mathrm{C}$ for 5 min then warmed to $\mathrm{rt}$ and stirred for an additional $1 \mathrm{~h}$. The precipitate was filtered off and the resulting filtrate was concentrated in vacuo. The crude material was purified by flash chromatography on silica gel column using 5\% ethyl acetate/hexanes as the eluant to afford hex-5-ynoic acid 1-methyl-prop-2ynyl ester as a yellow oil (1.77 g, 81\%).

To a stirred solution of 5-hexynoic acid 1-methy-prop-2-ynyl ester (700 mg, 4.27 mmol) in benzene $(4 \mathrm{~mL})$ were added triethylamine $(1.20 \mathrm{~mL}, 8.54 \mathrm{mmol})$ and triisopropylsilyl trifluoromethanesulfonate $(1.72 \mathrm{~mL}, 6.41 \mathrm{mmol})$. The solution was stirred at $\mathrm{rt}$ for $9 \mathrm{~h}$. The two layers were separated and the bottom layer was extracted with benzene. The benzene layers were combined and concentrated. The crude material was purified by flash column chromatography on silica gel using 5\% ethyl acetate/hexanes as the eluant to afford 2-(3-butynyl)-3,4-hexadienoic acid triisopropylsilyl ester ( $874 \mathrm{mg}, 2.73 \mathrm{mmol})$ as a colorless oil.

2-(3-Butynyl)-3,4-hexadienoic acid triisopropylsilyl ester ( $874 \mathrm{mg}, 2.73 \mathrm{mmol}$ ) was dissolved in ether $(6 \mathrm{~mL})$ and the solution was cooled to $0{ }^{\circ} \mathrm{C}$. To this solution was added lithium aluminum hydride $(75 \mathrm{mg}, 1.98 \mathrm{mmol})$. The reaction was stirred at $0{ }^{\circ} \mathrm{C}$ for $2 \mathrm{~h}$. Water $(75 \mu \mathrm{L}), 15 \% \mathrm{NaOH}(75 \mu \mathrm{L})$ and water $(225 \mu \mathrm{L})$ were added successively. The resulting white precipitate was filtered off and the filtrate was concentrated. The 
crude material was purified by flash chromatography using $15 \%$ ethyl acetate/hexanes as the eluant to afford title compound 29 as a colorless oil (295 mg, 72\%) : ${ }^{1} \mathrm{H}$ NMR (300 $\left.\mathrm{MHz}, \mathrm{CDCl}_{3}\right) \delta$ 5.18-5.08 (m, $\left.1 \mathrm{H}\right), 4.98-4.87(\mathrm{~m}, 1 \mathrm{H}), 3.59-3.48$ (m, $\left.2 \mathrm{H}\right)$, 2.41-2.14 (m, $3 \mathrm{H}), 1.99$ (bs, $1 \mathrm{H}), 1.93$ (t, $J=2.7 \mathrm{~Hz}, 1 \mathrm{H}), 1.75-1.68$ (m, $1 \mathrm{H}), 1.65$ (dd, $J=3.2,7.0$ $\mathrm{Hz}, 3 \mathrm{H}), 1.56-1.44(\mathrm{~m}, 1 \mathrm{H}) ;{ }^{13} \mathrm{C} \mathrm{NMR}\left(75 \mathrm{MHz}, \mathrm{CDCl}_{3}\right) \delta 205.1,90.4,86.7,84.1,68.4$, 65.6, 41.0, 29.8, 16.0, 14.4; IR (film) 3356, 3300, 2922, 2860, 2110, 1961, $1726 \mathrm{~cm}^{-1}$; EIHRMS calcd for $\mathrm{C}_{10} \mathrm{H}_{14} \mathrm{O}\left[\mathrm{M}^{+}\right] \mathrm{m} / \mathrm{z} 150.1045$, found 150.1039 .

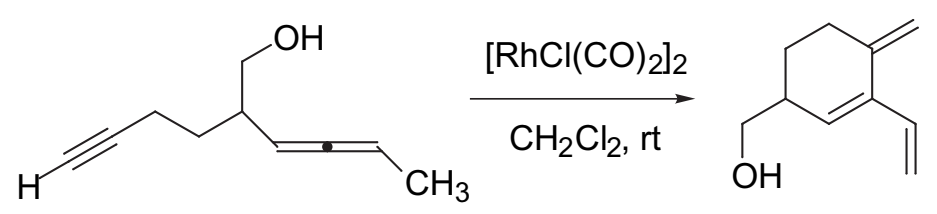

29

30

(4-Methylene-3-vinyl-cyclohex-2-enyl)-methanol (30). To a stirred solution of 2-(3-Butynyl)-3,4-hexadien-1-ol (29, $50 \mathrm{mg}, 0.33 \mathrm{mmol})$ in $2 \mathrm{~mL}$ of $\mathrm{CH}_{2} \mathrm{Cl}_{2}$ was added chlorodicarbonylrhodium (I) dimer $(6 \mathrm{mg}, 0.02 \mathrm{mmol})$. The reaction was stirred at $\mathrm{rt}$ for 3 h. After removal of $\mathrm{CH}_{2} \mathrm{Cl}_{2}$, the resulting crude material was purified by flash chromatography on silica gel using $5 \%$ ethyl acetate/hexanes as the eluant to afford 37 $\mathrm{mg}(80 \%)$ of $\mathbf{3 0}$ as a colorless oil : ${ }^{1} \mathrm{H} \mathrm{NMR}\left(300 \mathrm{MHz}, \mathrm{CDCl}_{3}\right) \delta 6.48(\mathrm{ddt}, J=1.0,10.8$, $17.2 \mathrm{~Hz}, 1 \mathrm{H}), 5.89$ (s, $1 \mathrm{H}), 5.41$ (dd, $J=1.9,17.3 \mathrm{~Hz}, 1 \mathrm{H}), 5.10$ (dd, $J=1.9,10.8 \mathrm{~Hz}, 1$ H), 5.03 (s, $1 \mathrm{H}), 4.89$ (s, $1 \mathrm{H}), 3.61$ (d, $J=5.8 \mathrm{~Hz}, 2 \mathrm{H}), 2.53-2.44(\mathrm{~m}, 2 \mathrm{H}), 2.40-2.30$ (m, $1 \mathrm{H}), 1.95-1.86(\mathrm{~m}, 1 \mathrm{H}), 1.55-1.43(\mathrm{~m}, 2 \mathrm{H}) ;{ }^{13} \mathrm{C} \mathrm{NMR}\left(75 \mathrm{MHz}, \mathrm{CDCl}_{3}\right) \delta 142.8$, 138.2, 135.8, 128.1, 115.2, 109.8, 66.6, 39.6, 30.9, 26.0; IR (film) 3329, 2935, 2863, 1629, 1440, $1046 \mathrm{~cm}^{-1}$; EI-HRMS calcd for $\mathrm{C}_{10} \mathrm{H}_{14} \mathrm{O}\left[\mathrm{M}^{+}\right] \mathrm{m} / \mathrm{z} 150.1045$, found 150.1045 .

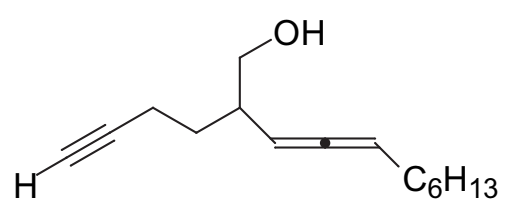

27

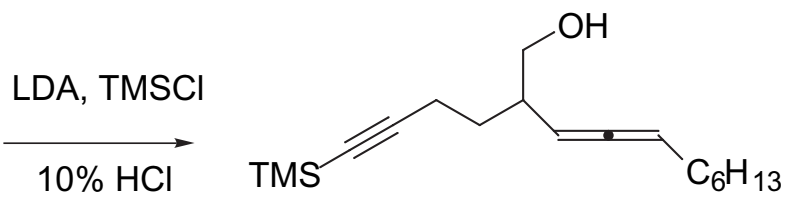

31 
2-(4-Trimethylsilyl-but-ynyl)-undeca-3,4-dien-1-ol (31). To a stirred solution of 2-(3-Butynyl)-3,4-undecadien-1-ol (27, $210 \mathrm{mg}, 0.96 \mathrm{mmol})$ in $3 \mathrm{~mL}$ of THF at $-78 \mathrm{C}$ was added $\mathrm{n}-\mathrm{BuLi}(2.5 \mathrm{M}, 0.80 \mathrm{~mL})$. The reaction was stirred for $10 \mathrm{~min}$. Then chlorotrimethylsilane $(0.37 \mathrm{~mL}, 2.9 \mathrm{mmol})$ was added. The reaction was stirred at $78^{\circ} \mathrm{C}$ for $50 \mathrm{~min}$, then the reaction was warmed to $\mathrm{rt}$ and stirred for another $30 \mathrm{~min} .10 \% \mathrm{HCl}$ solution ( $3 \mathrm{~mL}$ ) was added to the reaction solution and the mixture was stirred for $2 \mathrm{~h}$ at rt. The two layers were separated and the aqueous layer was extracted with ether. The organic layers were combined, dried $\left(\mathrm{Na}_{2} \mathrm{SO}_{4}\right)$ and concentrated under vacuo. The resulting residue was purified by column chromatography to afford of 2-(4-trimethylsilylbut-ynyl)-undeca-3,4-dien-1-ol (31) as a colorless oil (230 mg, 83\%): ${ }^{1} \mathrm{H}$ NMR (300 $\left.\mathrm{MHz}_{\mathrm{CDCl}}\right) \delta 5.192(\mathrm{dq}, J=2.0,6.6 \mathrm{~Hz}, 1 \mathrm{H}), 5.03-4.93(\mathrm{~m}, 1 \mathrm{H}), 3.66-3.50(\mathrm{~m}, 2 \mathrm{H})$, 2.40-2.23 (m, 3 H), 2.03-1.96 (m, 2 H), 1.72-1.52 (m, 3 H), 1.43-1.29 (m, 8 H), 0.89 (t, J $=6.6 \mathrm{~Hz}, 3 \mathrm{H}), 0.14(\mathrm{~s}, 9 \mathrm{H}) ;{ }^{13} \mathrm{C} \mathrm{NMR}\left(75 \mathrm{MHz}, \mathrm{CDCl}_{3}\right) \delta 204.4,107.1,92.4,91.2$, $84.8,65.8,41.4,31.7,30.2,29.1,29.0,28.8,22.6,17.6,14.0,0.1$; IR (film) 3338, 2960, 2923, 2167, $1956 \mathrm{~cm}^{-1}$; EI-HRMS calcd for $\mathrm{C}_{18} \mathrm{H}_{32} \mathrm{OSi}\left[\mathrm{M}^{+}\right] \mathrm{m} / \mathrm{z} 292.2222$, found 292.2209.

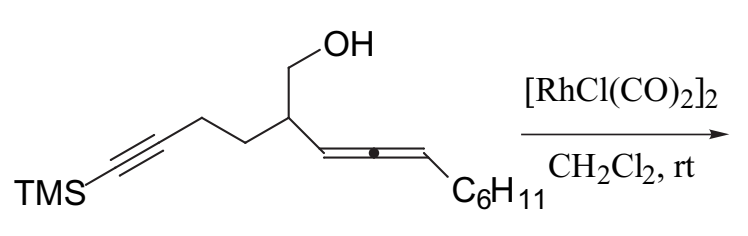

31

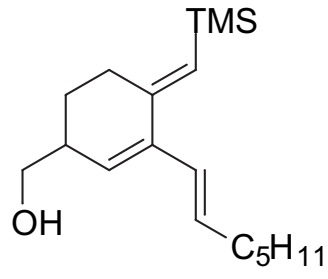

32

\section{(3-Hept-1-enyl-4-trimethylsilanylmethylene-cyclohex-2-enyl)-methanol (32).}

To a stirred solution of 2-(4-Trimethylsilyl-but-3-ynyl)-undeca-3,4-dien-1-ol (31, 70 mg, $0.24 \mathrm{mmol}$ ) in $2 \mathrm{~mL}$ of 1,2-dichloroethane was added chlorodicarbonylrhodium(I) dimer 
( $5 \mathrm{mg}, 0.01 \mathrm{mmol})$. The reaction was stirred at $\mathrm{rt}$ for $6 \mathrm{~h}$. After removal of 1,2dichloroethane, the resulting crude material was purified by flash chromatography on silica gel using 5\% ethyl acetate/hexanes as the eluant to afford $\mathbf{3 2}(48 \mathrm{mg}, 69 \%)$ as a 3:1 (determined by GC) mixture of $E$ and $Z$ isomers. The mixture was then subjected to HPLC to obtain 32-E and 32-Z in pure form.

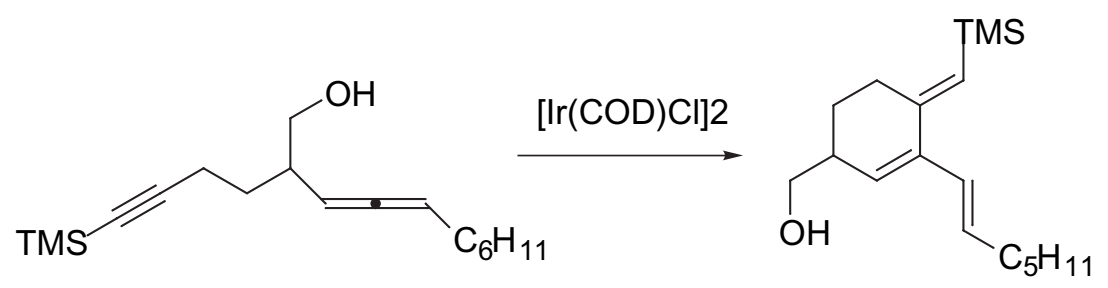

31

32

A stirred solution of 2-(4-Trimethylsilyl-but-3-ynyl)-undeca-3,4-dien-1-ol (31, $55 \mathrm{mg}, 0.19 \mathrm{mmol}$ ) in $2 \mathrm{~mL}$ of 1,2-dichloroethane was treated with chloro-1,5cyclooctadiene iridium(I) dimer (12 $\mathrm{mg}, 0.018 \mathrm{mmol})$. Then a solution of silver tetrafluoroborate $(0.05 \mathrm{M}$ in 1,2-dichloroethane, $0.72 \mathrm{~mL}, 0.036 \mathrm{mmol})$ was added dropwise. The reaction was monitored by GC and stirred for $2 \mathrm{~h}$ at $\mathrm{rt}$. The reaction solution was filter through a plug of silica gel and the resulting solution was concentrated to give 32-E as a pale yellow oil ( $41 \mathrm{mg}, 74 \%$ ). The $\mathrm{E}$ to $\mathrm{Z}$ ratio was determined by $\mathrm{GC}$ to be greater than $99: 1$.

(32-E: ${ }^{1} \mathrm{H}$ NMR $\left(300 \mathrm{MHz}, \mathrm{CDCl}_{3}\right) \delta 6.13(\mathrm{~d}, J=15.5 \mathrm{~Hz}, 1 \mathrm{H}), 5.82(\mathrm{~s}, 1 \mathrm{H})$; $5.81(\mathrm{dt}, J=6.8,15.5 \mathrm{~Hz}, 1 \mathrm{H}), 5.56(\mathrm{~s}, 1 \mathrm{H}), 2.61(\mathrm{ddd}, J=3.8,5.8,14.5 \mathrm{~Hz}, 1 \mathrm{H}), 2.55-$ $2.47(\mathrm{~m}, 1 \mathrm{H}), 2.38-2.28(\mathrm{~m}, 1 \mathrm{H}), 2.11(\mathrm{q}, J=7.0 \mathrm{~Hz}, 1 \mathrm{H}), 1.96-1.87$ (m, $1 \mathrm{H}), 1.53-$ $1.26(\mathrm{~m}, 8 \mathrm{H}), 0.90(\mathrm{t}, J=3.8 \mathrm{~Hz}, 3 \mathrm{H}), 0.1(\mathrm{~s}, 9 \mathrm{H}) ;{ }^{13} \mathrm{C} \mathrm{NMR}\left(75 \mathrm{MHz}, \mathrm{CDCl}_{3}\right) \delta 151.6$, 141.2, 132.3, 129.0, 127.1, 124.1, 66.8, 39.6, 32.9, 31.4, 30.1, 29.1, 26.3, 22.5, 14.0, 0.1; IR (film) 3389, 2916, 1665, $1244 \mathrm{~cm}^{-1}$. 
32-Z: ${ }^{1} \mathrm{H}$ NMR $\left(300 \mathrm{MHz}, \mathrm{CDCl}_{3}\right) \delta 5.98(\mathrm{~d}, J=11.4 \mathrm{~Hz}, 1 \mathrm{H}) ; 5.61(\mathrm{~s}, 1 \mathrm{H})$; $5.58(\mathrm{dt}, J=7.3,11.4 \mathrm{~Hz}, 1 \mathrm{H}) ; 5.48(\mathrm{~s}, 1 \mathrm{H}) ; 3.61(\mathrm{~d}, J=4.5 \mathrm{~Hz}, 2 \mathrm{H}) ; 2.63(\mathrm{ddd}, J=$ 3.8, 6.0, 14.5 Hz, $1 \mathrm{H}) ; 2.57-2.50(\mathrm{~m}, 1 \mathrm{H}) ; 2.40-2.29(\mathrm{~m}, 1 \mathrm{H}) ; 2.08(\mathrm{qd}, J=1.5,7.3 \mathrm{~Hz}$, $1 \mathrm{H}) ; 1.99-1.90(\mathrm{~m}, 1 \mathrm{H}) ; 1.57-1.45(\mathrm{~m}, 1 \mathrm{H}), 0.1(\mathrm{~s}, 9 \mathrm{H}) ;{ }^{13} \mathrm{C} \mathrm{NMR}\left(75 \mathrm{MHz}, \mathrm{CDCl}_{3}\right) \delta$ $151.3,137.9,133.0,129.5,128.2,124.6,66.8,39.3,31.5,29.7,29.5,28.4,26.1,22.5$, 14.1, 0.1; IR (film) 3353, 2953, 2924, $1571 \mathrm{~cm}^{-1}$. 